OPEN ACCESS

Edited by:

Francesca Trojsi,

Università degli Studi della Campania

"Luigi Vanvitelli" Naples, Italy

Reviewed by:

Yang $\mathrm{Hu}$,

Stanford University, United States

Antonio Giorgio,

University of Siena, Italy

Sandra Hanekamp,

Harvard University, United States

*Correspondence: Raffaele Nuzzi prof.nuzzi_raffaele@hotmail.it

Specialty section:

This article was submitted to

Neurodegeneration,

a section of the journal

Frontiers in Neuroscience

Received: 18 January 2018

Accepted: 09 May 2018

Published: 29 May 2018

Citation:

Nuzzi R, Dallorto L and Rolle T (2018)

Changes of Visual Pathway and Brain

Connectivity in Glaucoma: A

Systematic Review.

Front. Neurosci. 12:363.

doi: 10.3389/fnins.2018.00363

\section{Changes of Visual Pathway and Brain Connectivity in Glaucoma: A Systematic Review}

\author{
Raffaele Nuzzi ${ }^{*}$, Laura Dallorto and Teresa Rolle \\ Eye Clinic, Department of Surgical Sciences, University of Torino, Turin, Italy
}

Background: Glaucoma is a leading cause of irreversible blindness worldwide. The increasing interest in the involvement of the cortical visual pathway in glaucomatous patients is due to the implications in recent therapies, such as neuroprotection and neuroregeneration.

Objective: In this review, we outline the current understanding of brain structural, functional, and metabolic changes detected with the modern techniques of neuroimaging in glaucomatous subjects.

Methods: We screened MEDLINE, EMBASE, CINAHL, CENTRAL, LILACS, Trip Database, and NICE for original contributions published until 31 October 2017. Studies with at least six patients affected by any type of glaucoma were considered. We included studies using the following neuroimaging techniques: functional Magnetic Resonance Imaging (fMRI), resting-state fMRI (rs-fMRI), magnetic resonance spectroscopy (MRS), voxel- based Morphometry (VBM), surface-based Morphometry (SBM), diffusion tensor MRI (DTI).

Results: Over a total of 1,901 studies, 56 case series with a total of 2,381 patients were included. Evidence of neurodegenerative process in glaucomatous patients was found both within and beyond the visual system. Structural alterations in visual cortex (mainly reduced cortex thickness and volume) have been demonstrated with SBM and VBM; these changes were not limited to primary visual cortex but also involved association visual areas. Other brain regions, associated with visual function, demonstrated a certain grade of increased or decreased gray matter volume. Functional and metabolic abnormalities resulted within primary visual cortex in all studies with fMRI and MRS. Studies with rs-fMRI found disrupted connectivity between the primary and higher visual cortex and between visual cortex and associative visual areas in the task-free state of glaucomatous patients.

Conclusions: This review contributes to the better understanding of brain abnormalities in glaucoma. It may stimulate further speculation about brain plasticity at a later age and therapeutic strategies, such as the prevention of cortical degeneration in patients with glaucoma. Structural, functional, and metabolic neuroimaging methods provided evidence of changes throughout the visual pathway in glaucomatous patients. Other brain areas, not directly involved in the processing of visual information, also showed alterations.

Keywords: glaucoma, retinal ganglion cells, neurodegeneration, neuroplasticity, systematic review 


\section{INTRODUCTION}

\section{Rationale}

Glaucoma is a leading cause of irreversible blindness worldwide (Tham et al., 2014) and is characterized by the death of retinal ganglion cells (RGC) and their axons (Weinreb et al., 2014). The global incidence of primary open angle glaucoma (POAG) is anticipated to increase to 65 million by 2020 (Kapetanakis et al., 2016).

Numerous studies showed brain changes, especially in the visual pathway in glaucoma (Gupta and Yücel, 2003; Davis et al., 2016). The neurodegenerative process has been established in glaucomatous damage (Gupta et al., 2007; Chang and Goldberg, 2012). As a matter of fact, POAG presents important analogies with other neurodegenerative diseases: it has been shown that RGC share the same cell death mechanisms with Alzheimer's disease, AD (McKinnon, 2012). Protein misfolding is one of the identified mechanisms capable of triggering the apoptotic cascade in glaucomatous pathogenesis (Wostyn et al., 2009). Indeed, the $\beta$-amyloid deposits, characteristic of Alzheimer's disease, have recently been implicated in the pathogenesis of glaucoma (Wostyn et al., 2010). The incidence of glaucoma is increased in patients with $\mathrm{AD}$ compared to controls with the same age (Bayer et al., 2002; Tamura et al., 2006). Furthermore, the progression of visual field defects is accelerated in patients with open-angle glaucoma and $\mathrm{AD}$ compared to patients with open-angle glaucoma without AD (Bayer and Ferrari, 2002).

Moreover, nowadays, new treatments targeted visual pathway. Several neuroprotective strategies and drugs have been studied and some of them are used in the clinical practice. Neuroprotection consist in the prevention of neurons death (Jutley et al., 2017; Sena and Lindsley, 2017). Molecules that have passed through clinical test are memantine (NMDA glutamate receptor antagonist) and brimonidine (alpha2-adrenergic agonist). Neuroprotection is not effective for RGCs which have already been injured, thus neuroenhancement is also proposed as a therapeutic approach. Axon regeneration is the aim of neurotrophic factors such as nerve growth factor (NGF) and ciliary neurotrophic factor (CNTF). Human trials using these exogenous neurotrophic factors showed promising results but of limited duration and with the inconvenient of repeated injections. In recent years, stem cells have been widely studied as potential source of cell replacement. Several types and methods of administration (subtenionan, retrobulbar, and intravitreal) have been proposed and experimented in clinical trials even in humans. Another approach, still limited to murine examples, is the RGC transplantation. Preliminary data showed promising approach in retinas with degenerating RGCs (Venugopalan et al., 2016). Finally, retinal implants provide electrical stimulation of different targets but their clinical application is limited due to limited visual perception (Mathieson et al., 2012).

The efficacy of these modern approaches impose a deep knowledge of, cortical reorganization, neuroplasticity, and rearrangement of the visual pathways in glaucoma. Almost all approaches targeted RGCs of optic nerve require the integrity of posterior visual pathway or the adaptation of nervous system through plastic capacity. Moreover, new insights into brain changes in glaucoma may stimulate new therapeutic strategies.

The advent of non-invasive brain-imaging techniques has led to a rapid growth in studies investigating the brain damage in glaucomatous patients. Newer techniques and protocols enable the study of anatomical gray and white matter changes through structural techniques, brain connectivity and functional responses after stimulation through functional magnetic resonance imaging (fMRI) and changes in brain metabolite levels through metabolic technique (Fiedorowicz et al., 2011; Mastropasqua et al., 2015; Brown et al., 2016; Prins et al., 2016).

Structural brain techniques include diffusion MRI (diffusion tensor imaging, DTI) which can reveal abnormalities in white matter structure and brain connectivity through diffusivity of water molecules along the axons (Alexander et al., 2007). Voxel-based morphometry analysis (VBM) which is computed on MRI, quantifies the tissue concentration of gray and white matter volume (Ashburner and Friston, 2000). Surfacebased morphometry (SBM), throughT1-weighted morphometric analysis, provides data about the brain structure, such as thickness, curvature, and surface area of brain cortex (Clarkson et al., 2011).

Functional magnetic resonance imaging (fMRI) is a neuroimaging procedure capable to detect functional brain activities after a stimulus through detecting changes in blood flow (Miki et al., 2001, 2002; Kollias, 2004). It is also used to evaluate interactions between brain areas in a resting subject without any visual stimulus, the so-called "resting state fMRI" (Smitha et al., 2017). Finally, metabolic method such as the proton magnetic resonance spectroscopy (MRS) is able to detect and quantify certain biochemical compounds in brain tissue (Boucard et al., 2007).

\section{Objective}

This systematic review focuses on brain changes detected with the modern techniques of neuroimaging (structural, functional, and metabolic methods) in glaucomatous subjects.

\section{Research Question}

What have studies on neuroimaging in glaucomatous patients found thus far?

When do brain changes occur and what is the cause of these changes in the glaucomatous patients?

\section{METHODS}

We adopted the Preferred Items for Systematic Reviews and Meta-Analyses (PRISMA) guidelines.

\section{Search Strategy}

We searched: MEDLINE (Ovid), CENTRAL (which contains the Cochrane Eyes and Vision Group Trials Register), EMBASE (Ovid), Latin American and Caribbean Literature on Health Sciences (LILACS), CINAHL (EBSCO), Trip Database, and The National Institute for Health and Care Excellence (NICE). The construction of search strategies was performed using 
database specific subject headings and keywords. The MEDLINE search strategy was provided as Supplementary Material (Supplementary Data 1). These searches were supplemented by hand searching the bibliographies of all the included studies.

Gray literature was not considered. Accepted languages of publication were: English, German, French, Spanish, Portuguese and Italian. Articles published until October 31, 2017 were included.

\section{Study Design}

Randomized controlled trials (RCT), clinical trials, nonrandomized comparative studies, cohort studies, and case series (CS) were included.

Case report, case series with $<6$ patients and article with absence of outcome data were excluded.

\section{Participants, Interventions, Comparators}

We included studies on patients affected by POAG, primary angle closure glaucoma (PACG), and normal tension glaucoma (NTG).

Glaucomatous damage could be both unilateral and bilateral. Stages of glaucoma were not exclusion criteria.

We included studies using the following neuroimaging techniques:

- Diffusion tensor MRI (DT MRI/ DTI)

- Voxel- based Morphometry (VBM)

- Surface-based Morphometry (SBM)

- Functional Magnetic Resonance Imaging (fMRI)

- Resting-state fMRI (rs-fMRI)

- Magnetic resonance spectroscopy (MRS)

\section{Data Sources, Studies Sections, and Data Extraction}

According to the PRISMA flow diagram screening of titles and abstracts was carried out. Not pertinent articles were rejected. Duplicates were removed using EPPI reviewer (by EPPI-Center, Social Science Research Unit, the Institute of Education, the University of London, UK). After this initial selection, full texts were independently judged for eligibility.

\section{Data Analysis}

The main outcome of this systematic review was the current understanding of brain changes in glaucomatous subjects. Structural, functional, and metabolic neuroimaging results were separately analyzed and presented, but their interaction was nonetheless studied. Differences between various types of glaucoma and various stages of disease were analyzed.

Specifically, characterizing parameters of each neuroimaging method are listed below:

- DTI: fractional anisotropy (FA), mean diffusivity (MD), and radial diffusivity $(\mathrm{RD})$. They all are measures of white matter damage.

- VBM: gray matter and white matter volume.

- SBM: thickness, curvature, and surface area of brain cortex.

- fMRI: Blood oxygenation level dependent (BOLD) evaluation. It detects changes in the blood flow of local brain regions through changes in hemoglobin and unoxygenated hemoglobin in the blood. In the manuscript we referred to fMRI for "task-fMRI," which measures responses during visual tasking

- rs-fMRI: Voxel-wise degree centrality (DC), that is the direct connections for a given voxel in the voxel-wise connectome, and functional connectivity (FC) between areas in resting conditions, without any visual tasks

- MRS: concentrations of the metabolites such as Nacetylaspartate (NAA), creatinine (Cr), and Choline (Cho)

\section{RESULTS}

\section{Study Selection}

A total of 1,901 studies were screened using the described search strategy. At the end of the selection process, 56 case series were included in the systematic review. Prisma flow diagram (Figure 1) gives details on screening process.

Thirty-one out of 56 (55\%) studies examined brain changes in terms of structural parameters (19 studies used DTI, 6 studies VBM, 4 studies SBM, one single study both VBM and SBM, and one single study both VBM and DTI). Nineteen out of fiftysix studies, (34\%) used functional techniques to evaluate brain changes following glaucomatous degeneration (10 studies used the fMRI and 9 studies the rs-fMRI). Metabolic methods (MRS) are used in 2 studies (4\%). Four studies (7\%) used both structural and functional techniques on the same group of patients: three studies used rs-fMRI, VBM, and DTI, one study used VBM and fMRI.

\section{Study Characteristics}

A total of 1,326 patient and 1,055 controls were considered in this systematic review. Forty-nine studies included POAG patient (41 studies only POAG patients, 7 studies included both POAG and NTG, one single study had both POAG and PACG). A total of 1,061 patient, corresponding to $80 \%$ of the total number had POAG. 154 out of 1326 (12\%) patients were affected by PACG (they are included in seven studies, one of these studies included both POAG and PACG patients). NTG was the less studied type, corresponding to $8 \%$ (111 patients) of the total. Eight studies included NTG patients, 7 studies had both NTG and POAG patients, one single study had NTG patients).

Patients characteristics of included studies are reported in Table 1. Patients included in this review show high heterogeneity in mean age ranging from 25 (Dai et al., 2013) to 76 (Bogorodzki et al., 2014) years. Severity of glaucoma damage varies from early to severe and some authors included different stages in the same study. Method of severity classification was not consistent throughout all studies, even though the most used is the HodappParrish-Anderson (HPA) classification.

\section{Outcomes of Structural Analysis}

Results of studies analyzing structural brain changes in glaucomatous patients are reported in Table 2.

Diffusion tensor imaging methodology has been extensively used to investigate differences in white matter tracts of visual pathway within glaucoma (19 out of 56 studies, $34 \%$ of all 


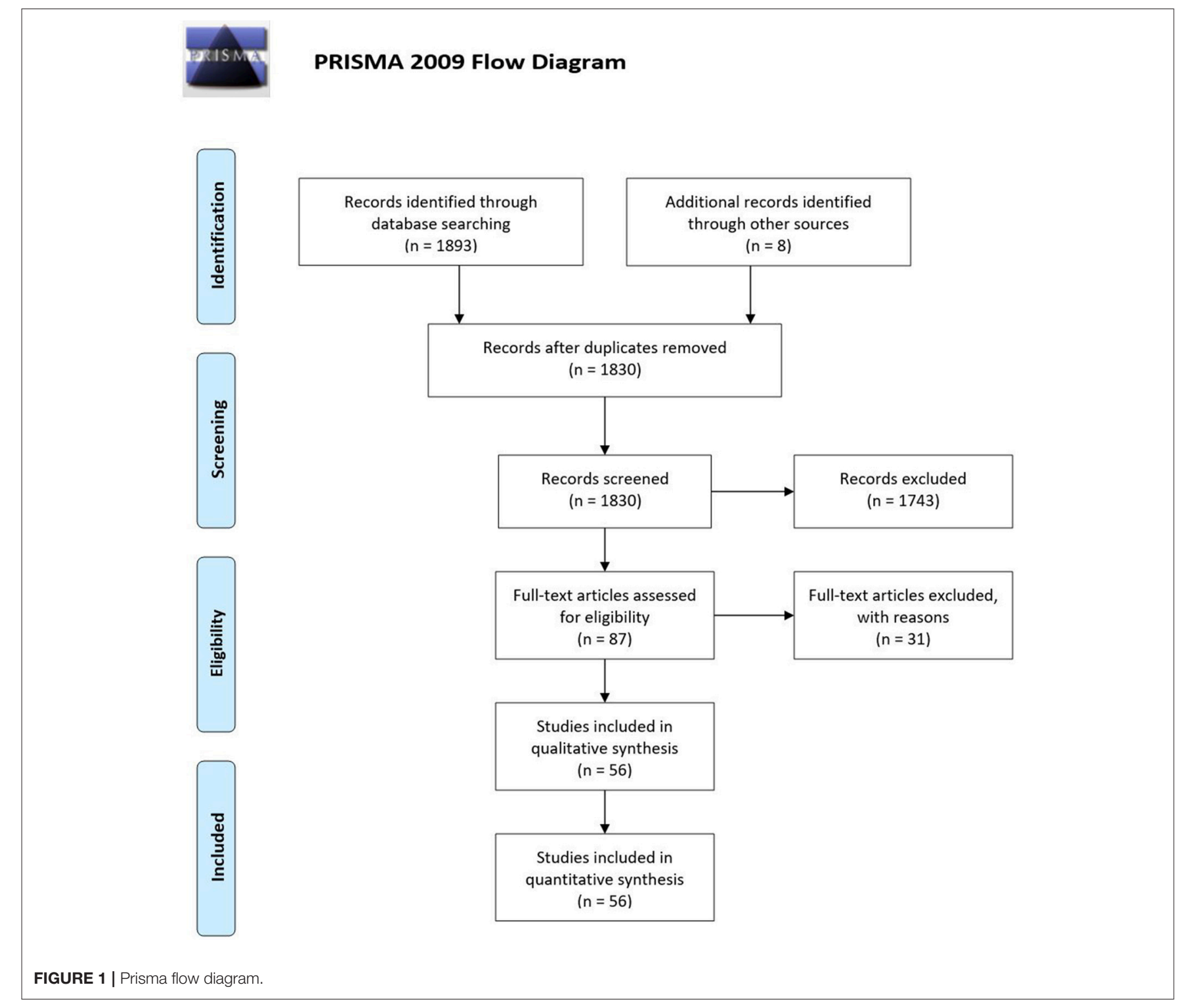

included studies). Decreased fractional anisotropy (FA) and increased mean diffusivity (MD) and radial diffusivity (RD) are sign of axon injury. Differences of microstructure in white matter structures have been found in the optic nerve, optic tracts, optic chiasm, optic radiations, and occipital lobe. These results were obtained in studies including POAG (Garaci et al., 2009; ElRafei et al., 2011, 2013; Engelhorn et al., 2011; Bolacchi et al., 2012; Nucci et al., 2012; Zhang et al., 2012; Zikou et al., 2012; Chen Z. et al., 2013; Lu et al., 2013; Michelson et al., 2013; Frezzotti et al., 2014, 2016; Kaushik et al., 2014; Omodaka et al., 2014; Schoemann et al., 2014; Sidek et al., 2014; Murai et al., 2015; Tellouck et al., 2016; Zhou et al., 2017a; Giorgio et al., 2018), NTG (Boucard et al., 2016) and PACG (Wang et al., 2013) patients.

Some of the studies using DTI methodology found alterations of white matter integrity not limited to the primary visual pathway. Boucard et al. (2016) revealed damage of corpus callosum and parietal lobe in NTG, Frezzotti et al. (2016) found alterations in the longitudinal fascicle, supramarginal gyrus, and superior parietal lobule of early stage open angle glaucoma. In addition, according to Giorgio et al study (Giorgio et al., 2018), superior longitudinal fascicle, white matter adjacent to precuneus, inferior frontal gyrus, and superior parietal lobe had microstructural alterations in both normal tension and open angle glaucoma compared to healthy subjects (Giorgio et al., 2018). Such white matter structures are related to higher aspects of visual and cognitive process. Furthermore, significant decreased FA was found in glaucomatous compared to healthy subjects in the inferior fronto-occipital fasciculus (implied in visuospatial function), longitudinal and inferior frontal fasciculi (related to visual memory), putamen, caudate nucleus, anterior, and posterior thalamic radiations and anterior and posterior limbs of the internal capsule (implied in direction, color, and orientation; Zikou et al., 2012). 


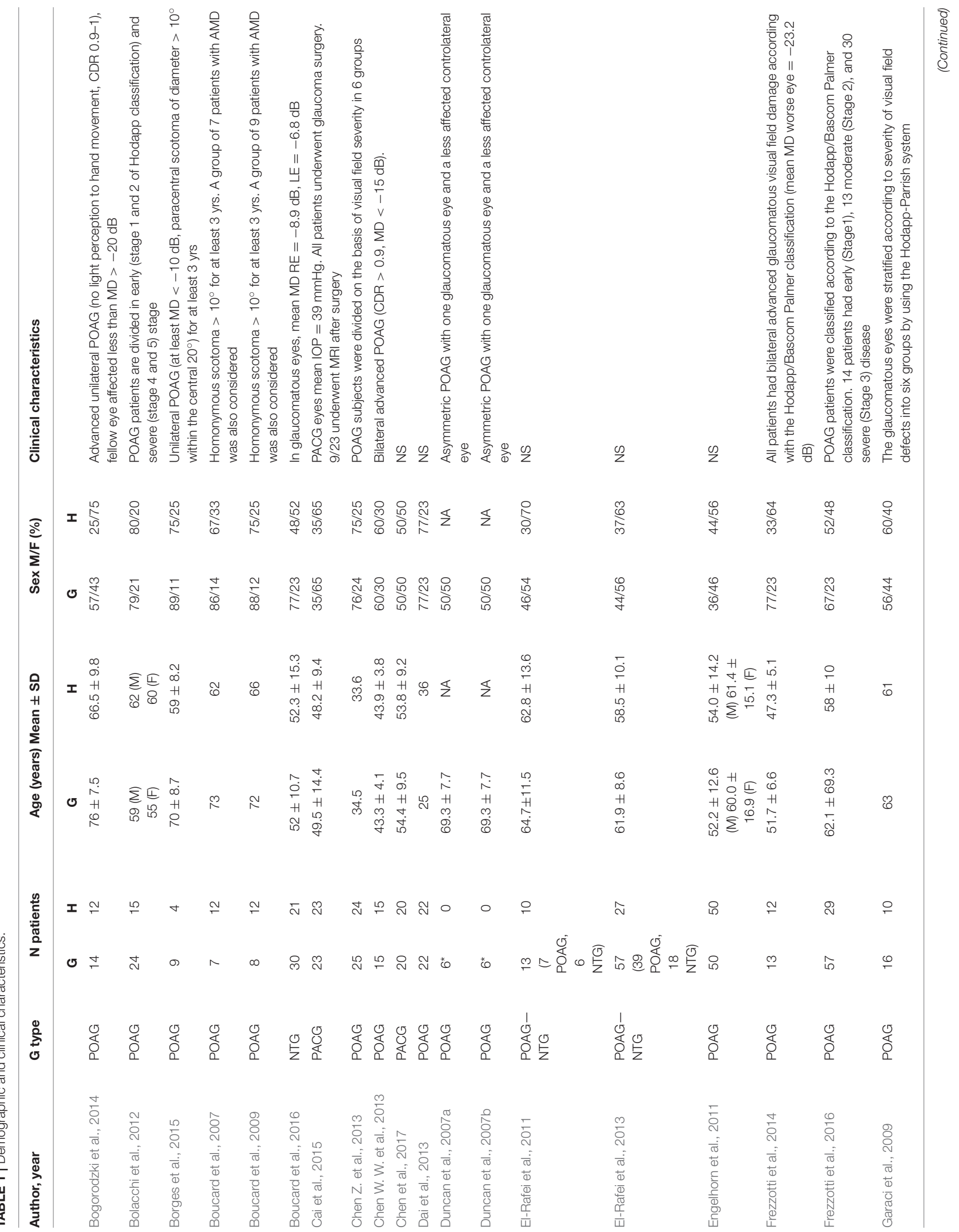




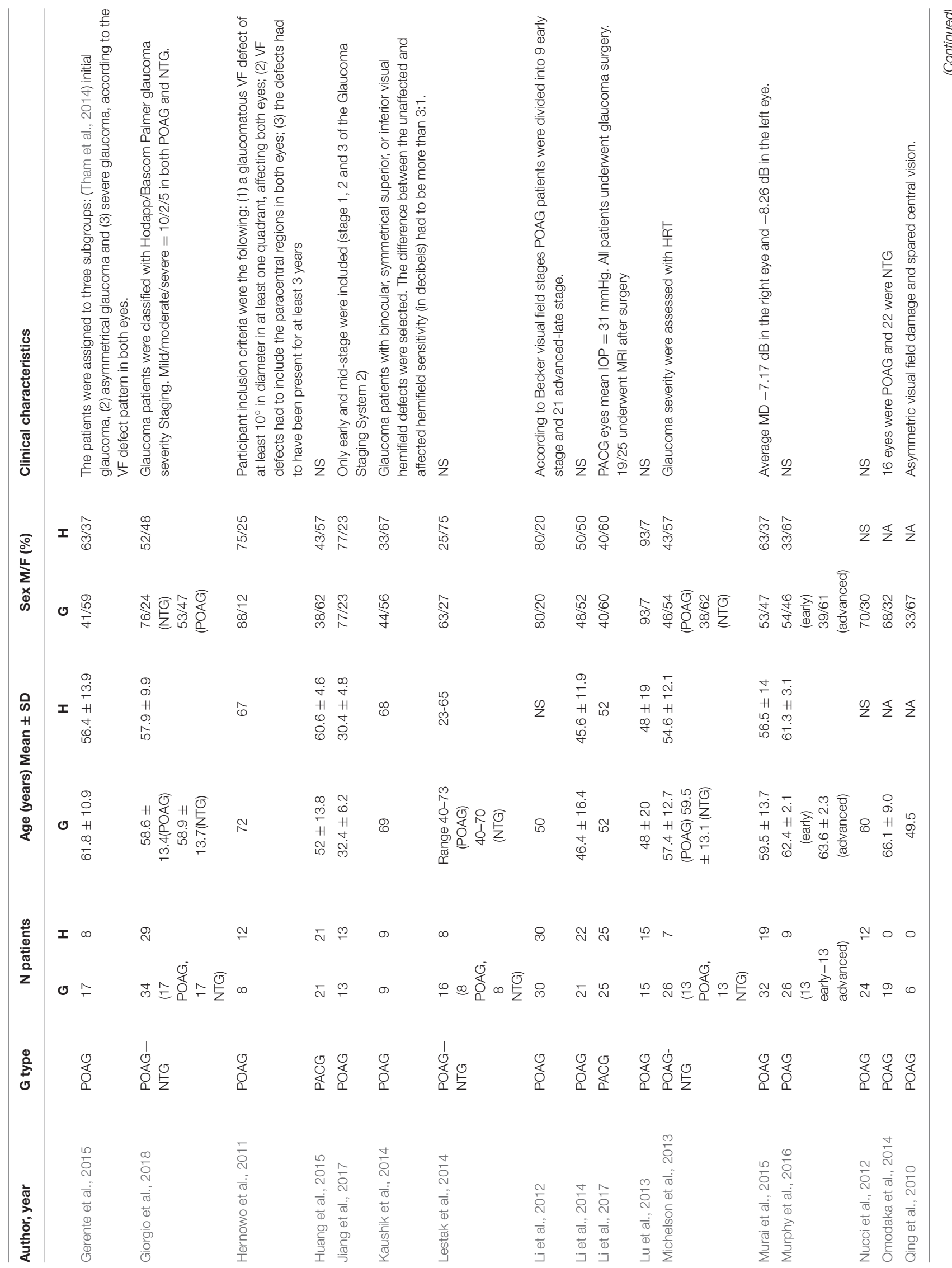




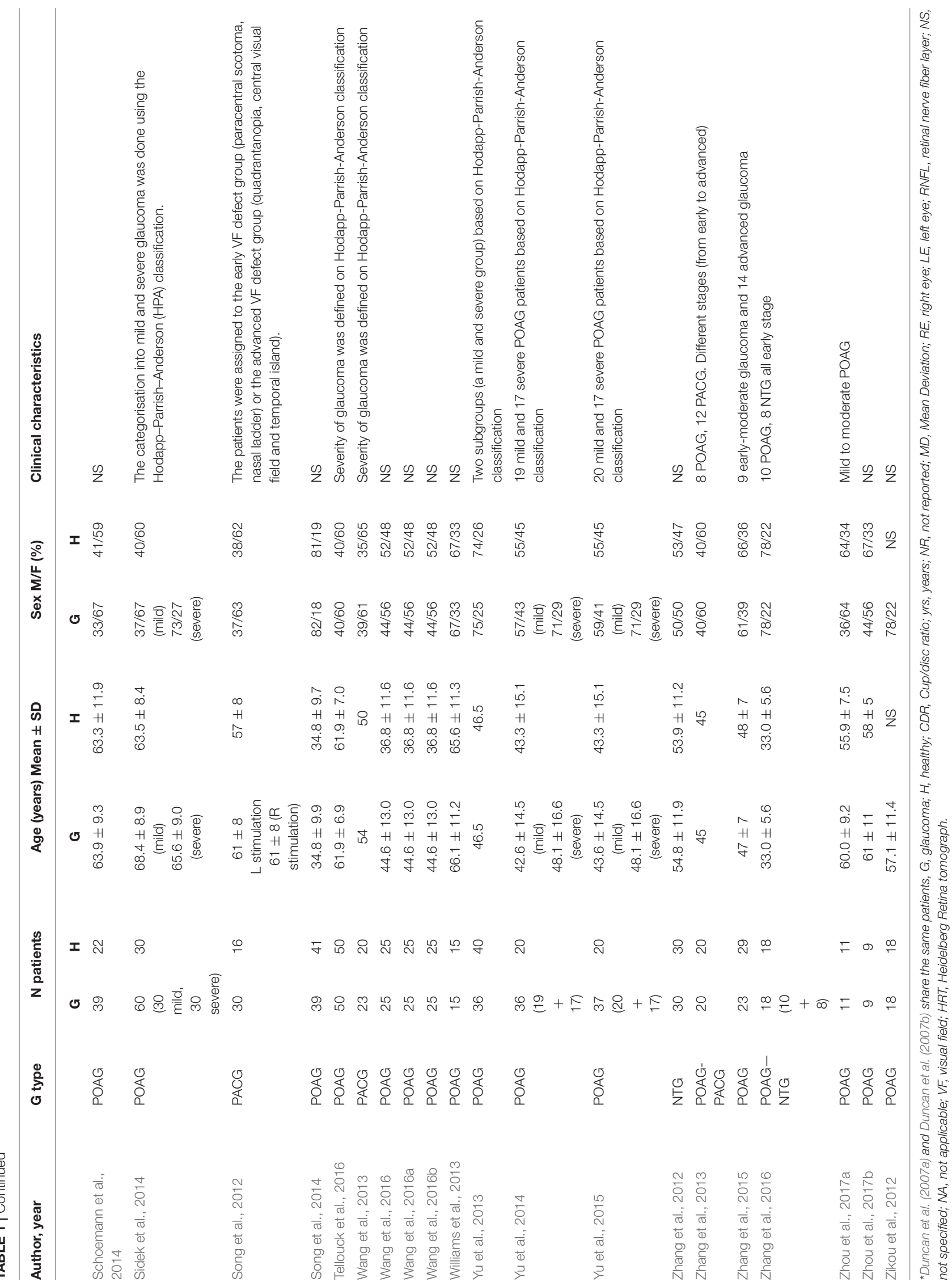




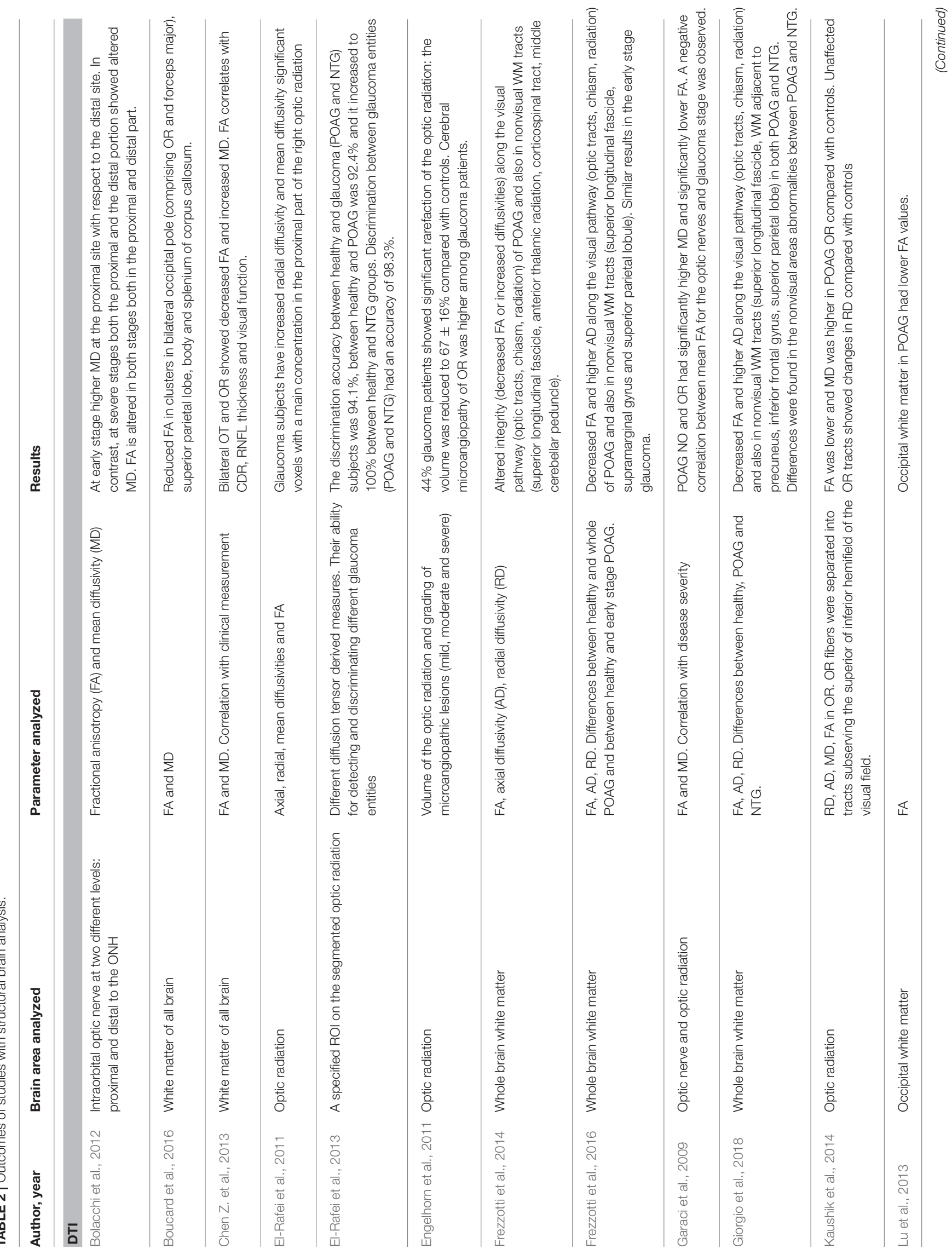




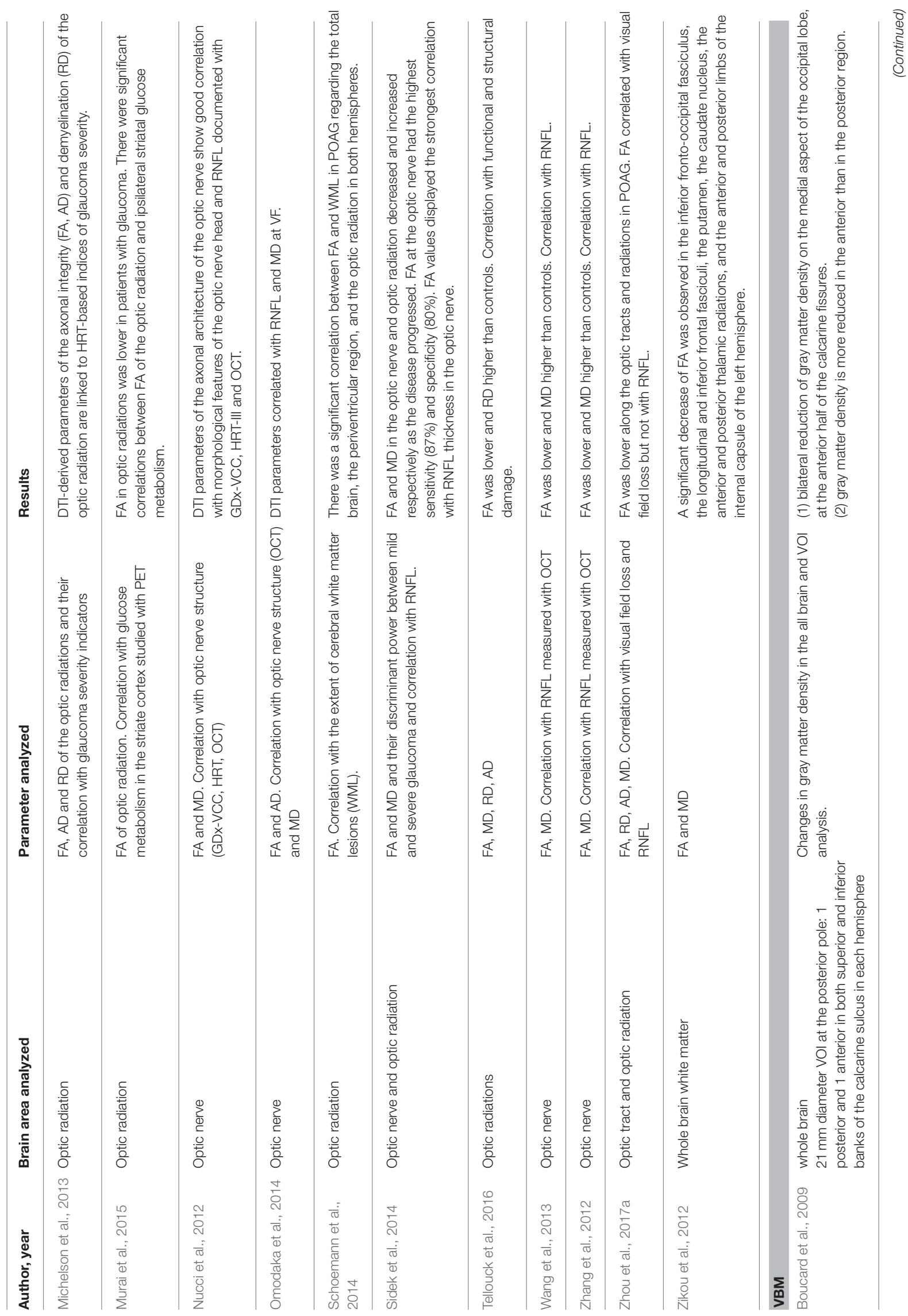



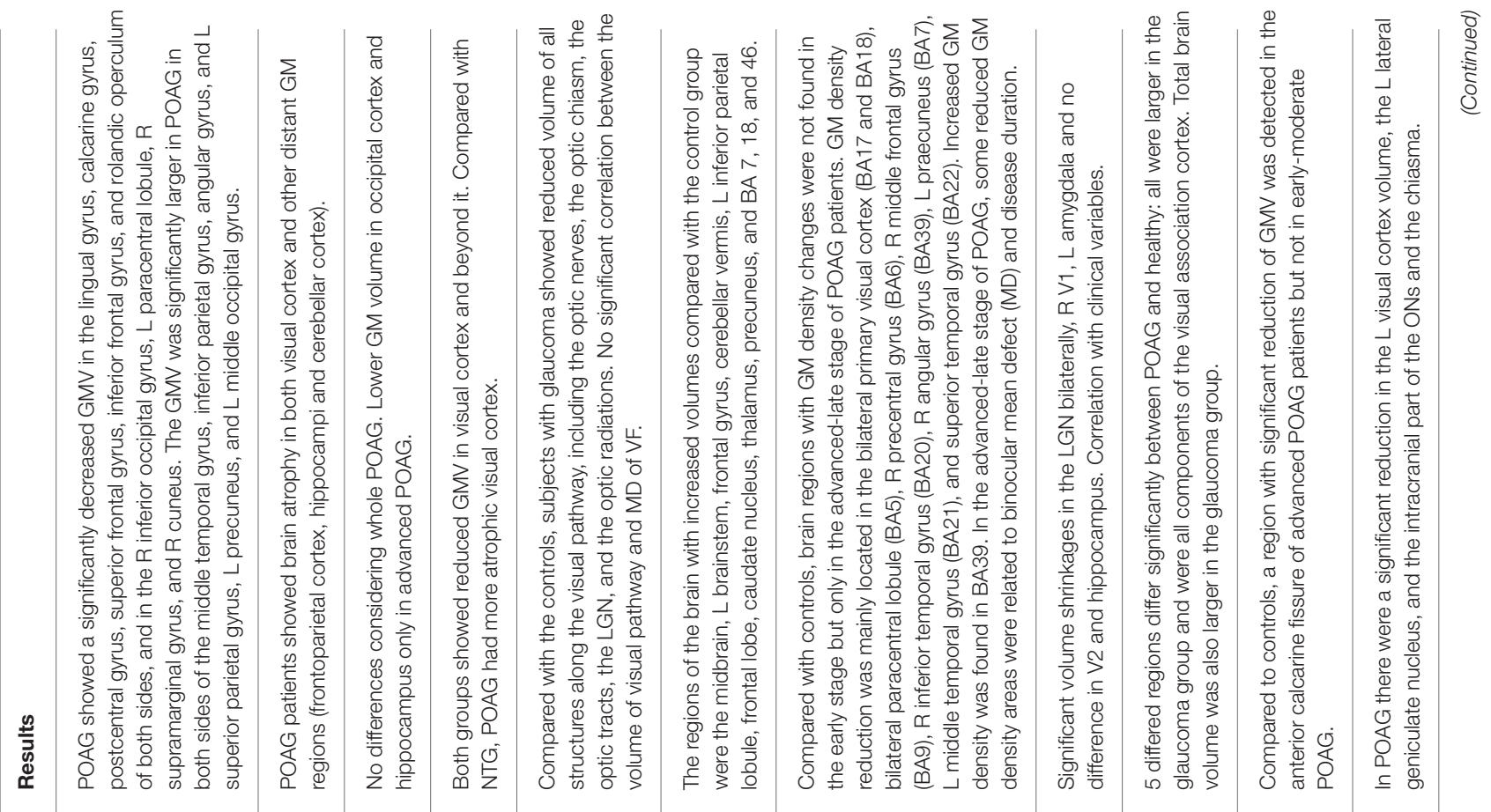
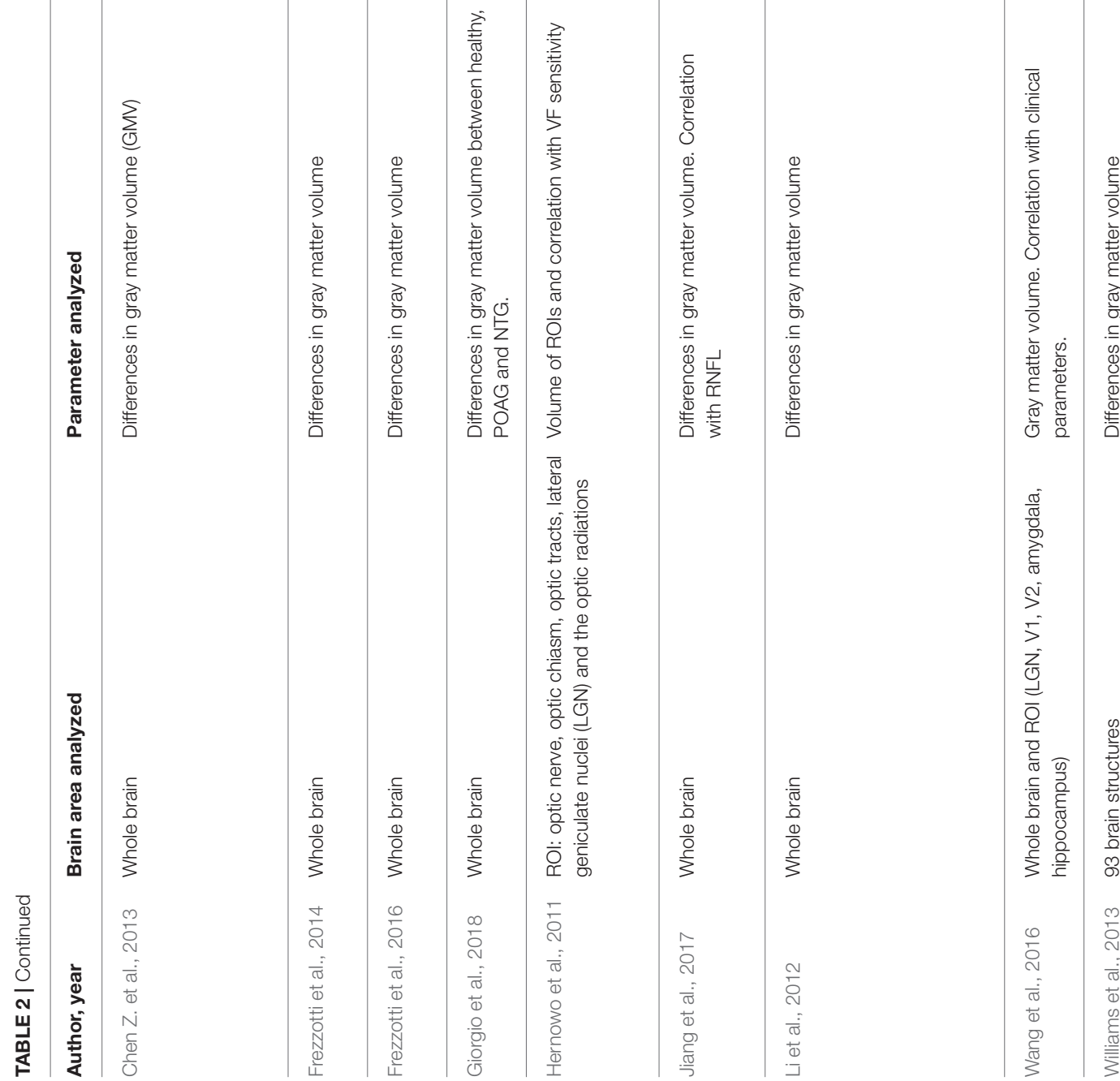


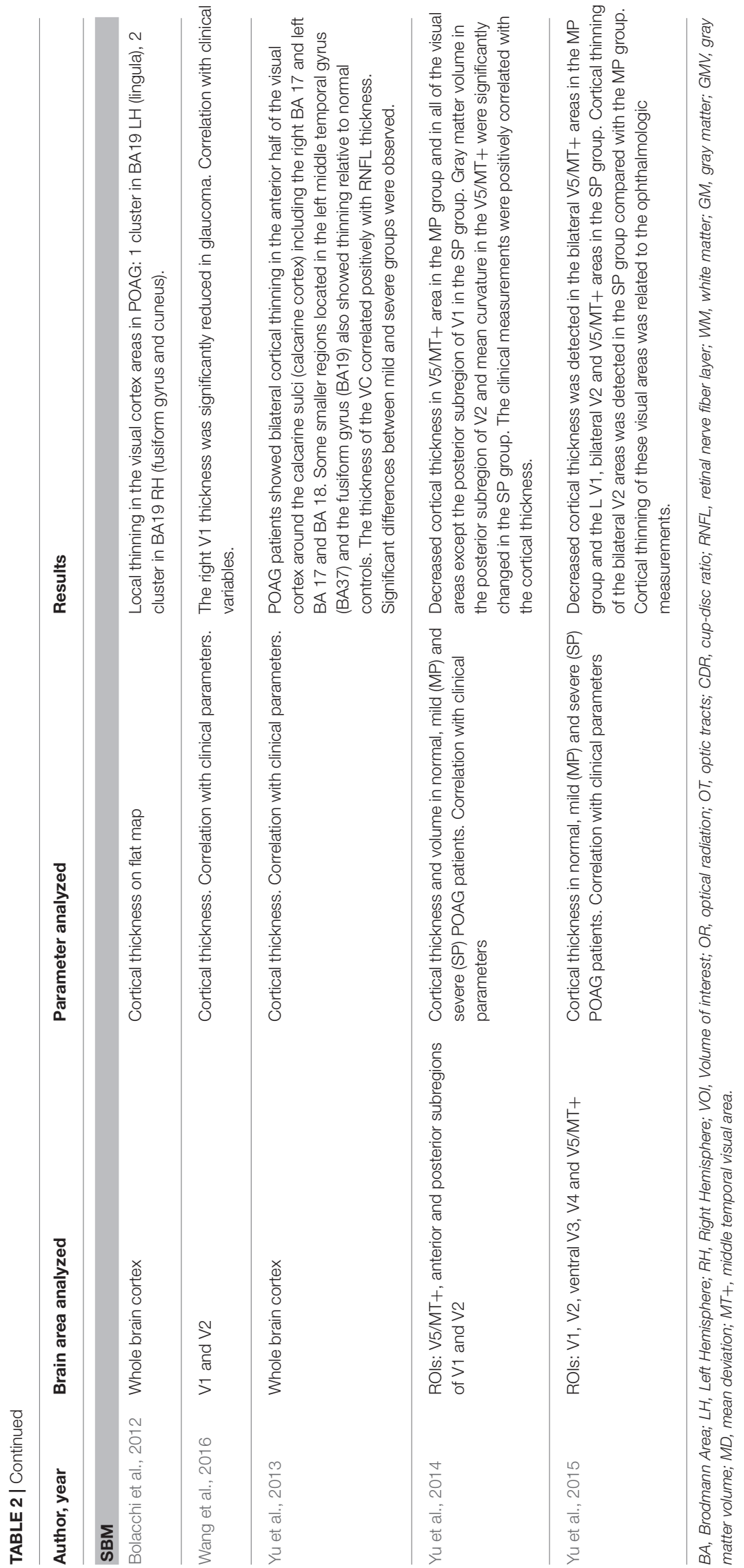


Dependence on glaucoma severity of changes in white matter tissue studied with DTI was demonstrated. Glaucoma severity showed a negative correlation with FA, and a positive correlation with $\mathrm{MD}$ and $\mathrm{RD}$ values of white matter tracts involved in the visual process (Garaci et al., 2009; Nucci et al., 2012; Zhang et al., 2012; Chen Z. et al., 2013; Michelson et al., 2013; Wang et al., 2013; Omodaka et al., 2014; Sidek et al., 2014; Tellouck et al., 2016; Zhou et al., 2017a). Correlation was found between DTI parameters and RNFL, mean deviation of visual field, cup-to-disc ratio.

Significant gray matter reduction within the primary visual cortex emerged from many studies using VBM (Hernowo et al., 2011; Li et al., 2012; Zikou et al., 2012; Chen W. W. et al., 2013; Williams et al., 2013; Frezzotti et al., 2014, 2016; Zhang et al., 2015; Wang et al., 2016; Jiang et al., 2017; Giorgio et al., 2018). Specifically, a significant reduction was found only in the anterior part of occipital pole (Boucard et al., 2007) strengthen the idea of retinotopic damage. Other studies showed clusters of gray matter reduction in glaucoma patients in areas other than occipital pole, including the lingual gyrus, calcarine gyrus, postcentral gyrus, superior frontal gyrus, inferior frontal gyrus, rolandic operculum, cerebellar cortex, and hippocampi (Li et al., 2012; Chen W. W. et al., 2013; Frezzotti et al., 2014).

The extent of gray matter atrophy was dependent on the level of glaucoma severity (Li et al., 2012; Zhang et al., 2015; Wang et al., 2016). Indeed, Frezzotti et al found lower gray matter volume only in advanced POAG (Frezzotti et al., 2016).

Moreover, gray matter was increased in some brain areas (middle temporal gyrus, inferior parietal gyrus, angular gyrus, midbrain, brainstem, frontal gyrus, cerebellar vermis, thalamus) of glaucomatous patients (Li et al., 2012; Chen W. W. et al., 2013; Williams et al., 2013; Jiang et al., 2017). All these brain regions involved were associated with visual functions.

Alterations in visual cortex has been demonstrated also with SBM. In particular, the five studies using SBM demonstrated a thinning in the visual cortex of POAG (Bolacchi et al., 2012; Yu et al., 2013, 2014, 2015; Wang et al., 2016). Reduced cortex thickness was not limited to primary visual cortex, but also involved association visual areas, such as lingula, fusiform gyrus, cuneus, middle temporal gyrus (Bolacchi et al., 2012; Yu et al., 2013, 2014, 2015). Positive correlation was found between thinning of visual cortex and clinical measurements ( $\mathrm{Yu}$ et al., 2013, 2014, 2015; Wang et al., 2016). Correlation with glaucoma severity was also demonstrated by significant differences of visual cortex thickness between mild and severe glaucoma groups, with increased damage in late-stage glaucoma (Yu et al., 2013).

\section{Outcomes of Functional and Metabolic Analysis}

Studies reporting functional and metabolic activity in glaucomatous patients are reported in Table 3.

Functional activity resulted greatly reduced within primary visual cortex after visual input from the glaucomatous eye in all studies using fMRI both in POAG (Duncan et al., 2007a; Qing et al., 2010; El-Rafei et al., 2013; Lestak et al., 2014; Borges et al., 2015; Gerente et al., 2015; Murphy et al., 2016; Zhang et al., 2016; Jiang et al., 2017; Zhou et al., 2017b), NTG (El-Rafei et al., 2013;
Lestak et al., 2014; Zhang et al., 2016) and PACG patients (Song et al., 2012).

Resting state fMRI (Rs-fMRI) is a tool for studying the human brain functional connectivity in resting state, without visual stimulation (Biswal et al., 2010). Voxel-wise degree centrality (DC) represents the number of connections for a given voxel and it, thus, quantifies the ability for information integration. The decreased connectivity was found in areas related to vision and also in other networks related to working memory and attention in POAG patients (Dai et al., 2013; Frezzotti et al., 2014, 2016; Chen et al., 2017; Giorgio et al., 2018). Decreased functional connectivity even without visual tasking was also observed in the bilateral visual cortices of PACG patients (Boucard et al., 2016).

Metabolic neuronal dysfunction was studied through quantification of certain biochemical compounds with proton MRS in two studies. The main brain metabolites studied by MRS included $\mathrm{N}$-acetylaspartate (NAA), Choline (Cho), and Creatine (Cr). NAA is localized within neurons and a decreased in NAA concentration is a sign of neuronal loss. Choline is a marker of membrane integrity, it decreases when a damage of neurons occurred. Creatinine, implied in energy metabolism, should remain constant throughout the brain even in neurodegenerative disease. Its concentration is used to calculate metabolic ratio such as NAA:Cr and Cho:Cr. Results of the two studies using MRS were contradictory, Boucard et al (Boucard et al., 2007) found no significant differences in the brain metabolites between the patients and the control group. On the contrary, Zhang et al. (2013), found decreased brain metabolites which are marker for neuronal integrity in geniculo-calcarine and striate area of occipital pole.

\section{DISCUSSION}

Studies of glaucomatous degeneration in the brain were initially based on experiments conducted on monkey who were induced glaucoma. The studies identified degeneration at the lateral geniculate nucleus (LGN) and primary visual cortex (V1) in response to increased IOP and optic nerve damage (Yücel et al., 2000, 2001). These results were supported by the same results in post-mortem human studies that reported a significant neurodegeneration in optic nerve, LGN and visual cortex in a glaucoma patient (Gupta et al., 2006). The advent of non-invasive brain imaging techniques has led to an increase in studies investigating the involvement of the brain in glaucoma pathology. The detection of brain neurodegeneration in glaucomatous subjects could open new future frontiers. As an example, innovative brain imaging methods have been proposed in the early detection of the disease and in the evaluation of therapeutic efficacy of novel neuroprotective strategies (Brown et al., 2016). This systematic review is aimed at bringing together different results deriving from other research regarding brain involvement in patients with glaucoma.

\section{Integration of Structural and Functional Results}

Studying brain changes in glaucoma patients both structurally, functionally, and metabolically provides for more information. 


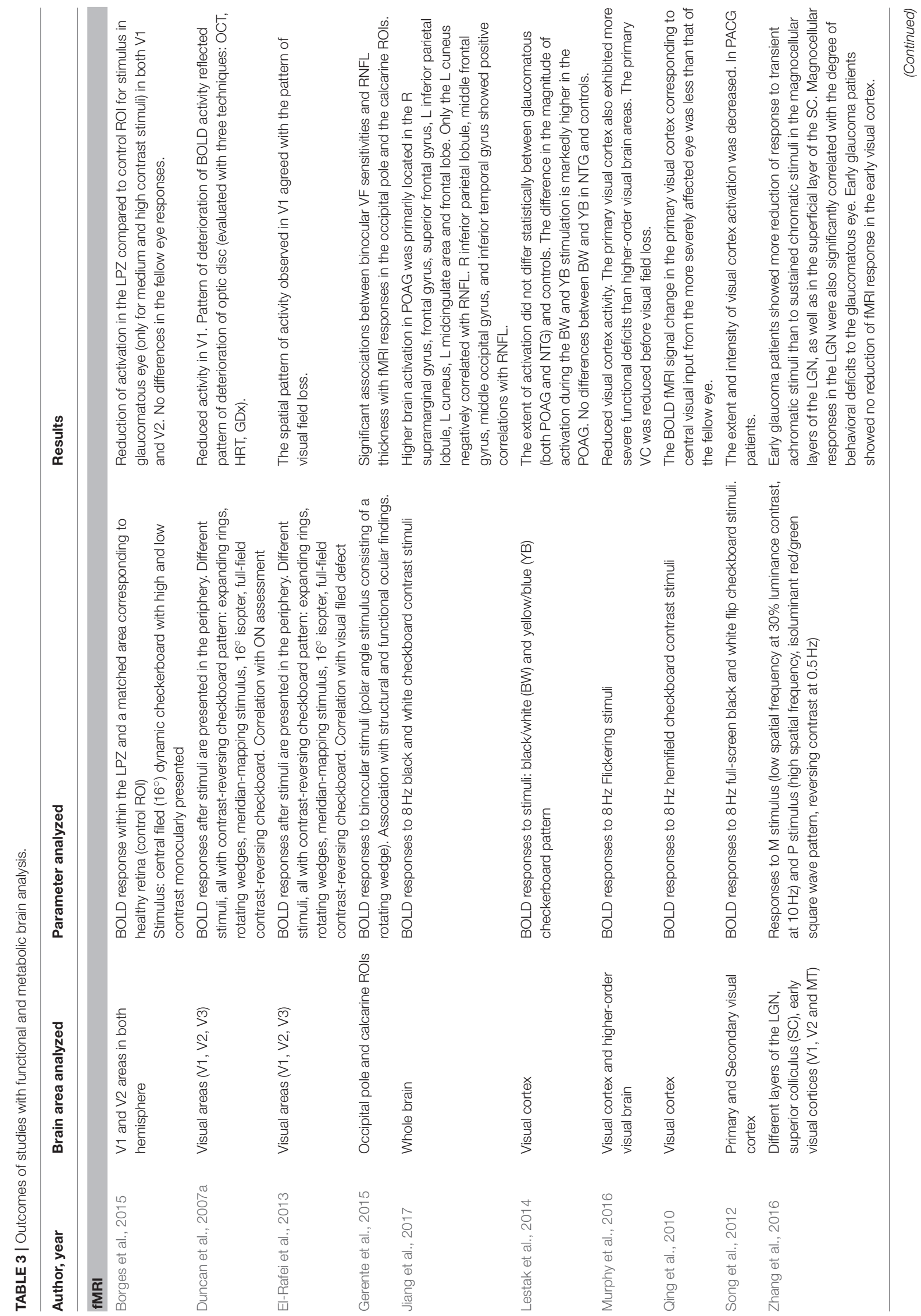




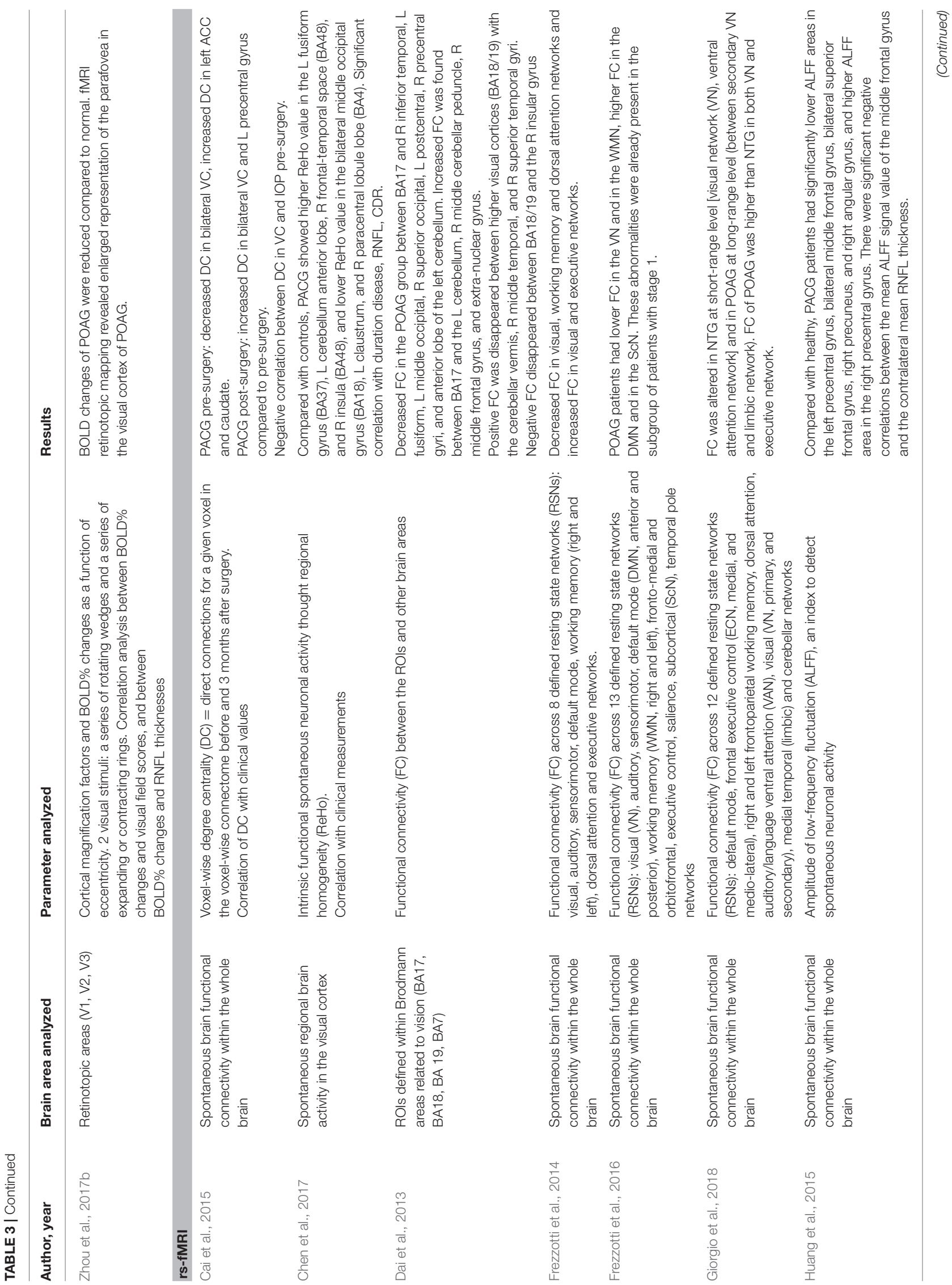




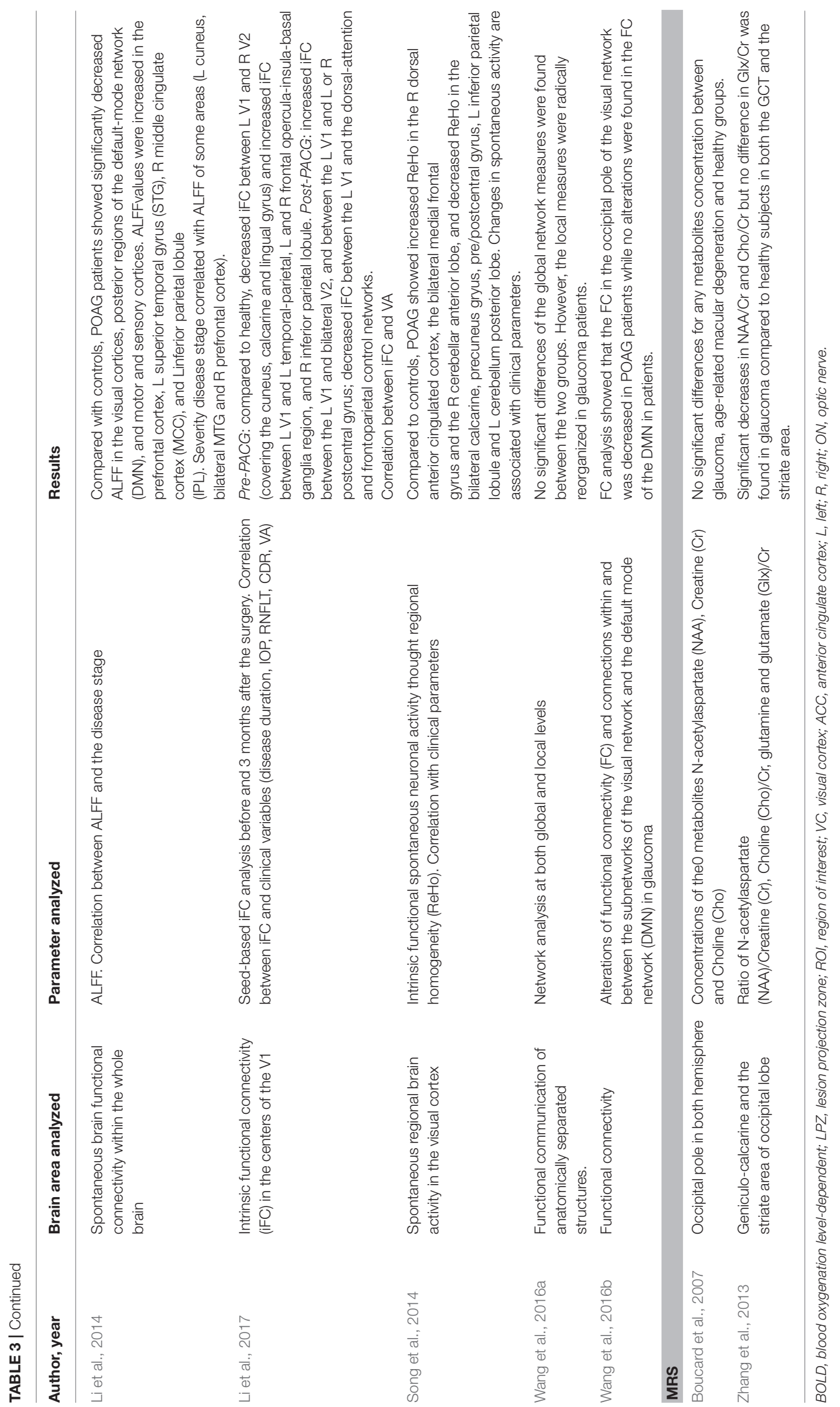




\section{White Matter of Visual Pathway (NO, NT, OR)}

Many studies using DTI demonstrated glaucomatous degeneration of the optic nerve (Garaci et al., 2009; Bolacchi et al., 2012; Nucci et al., 2012; Zhang et al., 2012; Wang et al., 2013; Omodaka et al., 2014), optic tract (Chen Z. et al., 2013; Frezzotti et al., 2014, 2016; Kaushik et al., 2014; Zhou et al., 2017a; Giorgio et al., 2018), and optic radiation (Garaci et al., 2009; El-Rafei et al., 2011, 2013; Engelhorn et al., 2011; Hernowo et al., 2011; Bolacchi et al., 2012; Chen Z. et al., 2013; Lu et al., 2013; Michelson et al., 2013; Frezzotti et al., 2014, 2016; Kaushik et al., 2014; Schoemann et al., 2014; Sidek et al., 2014; Gerente et al., 2015; Huang et al., 2015; Murai et al., 2015; Tellouck et al., 2016; Jiang et al., 2017; Zhou et al., 2017a; Giorgio et al., 2018). These findings are corroborated by other techniques, such as VBM. Subjects with glaucoma exhibited significant reductions in the volume of the visual pathway, including the optic nerves, chiasm, tracts, LGN, and optic radiations (Hernowo et al., 2011).

El-Rafei et al. demonstrated the ability of DTI- derived measures to differentiate between glaucomatous patients and healthy subjects but also between different glaucoma types (POAG and NTG) with an accuracy of $98.3 \%$ (El-Rafei et al., 2013).

According to Engelhorn's study (Engelhorn et al., 2011), rarefaction of optic radiation correlated both with the extent of optic nerve atrophy and with the extent of visual field defect. Thus, degeneration of optic radiation seems to be the result of anterograde transneuronal degeneration. Interestingly, the same study demonstrated a high prevalence $(80 \%)$ of microangiopathic lesions within the optic radiation supporting the concept of retrograde degeneration of RGCs starting from the optic radiation. Moreover, axonal impairment of optic radiation studied with DTI demonstrated good correlation with cerebral glucose hypometabolism in the striate cortex of POAG patients (Murai et al., 2015).

\section{Primary Visual Area}

In regard to primary visual cortex (V1), loss of gray matter at SBM (Bolacchi et al., 2012; Yu et al., 2013, 2014, 2015; Wang et al., 2016) and VBM (Boucard et al., 2009; Li et al., 2012; Zikou et al., 2012; Chen W. W. et al., 2013; Frezzotti et al., 2014; Zhang et al., 2015; Giorgio et al., 2018) analysis is consistent with a reduction of function proved by fMRI studies (Duncan et al., 2007a; Qing et al., 2010; Song et al., 2012; El-Rafei et al., 2013; Borges et al., 2015; Gerente et al., 2015; Murphy et al., 2016; Zhang et al., 2016; Jiang et al., 2017; Zhou et al., 2017b).

Using VBM, Boucard et al. (2007) showed a retinotopic specific gray matter reduction in primary visual cortex. Indeed, POAG subjects showed a reduction in gray matter density mainly in the anterior part of occipital pole, while AMD patients showed a reduction located more posteriorly in occipital cortex, in correspondence with the location of the foveal representation in visual cortex.

Nevertheless, the study of Boucard et al. (2007) investigating the concentrations of metabolites such as $\mathrm{N}$-acetylaspartate (NAA) in occipital pole using MRS, showed no alterations in patients with POAG compared to healthy subject. This could be explained with either absence of occipital degeneration or degeneration occurring with a slow rate, undetectable with MRS. The MRS method presents a limitation: it only measures reduced NAA levels when the degenerative process is currently taking place.

Interestingly, the deterioration of cortical activity and structure in V1 correlated with structural measurement of optic disc. Structural analysis of optic disc damage was conducted with scanning laser polarimetry with variable corneal compensation (GDx-VCC), confocal scanning laser ophthalmoscopy (SLO), and optical coherence tomography (OCT). In addition, functional activity of $\mathrm{V} 1$ is altered in a manner consistent with the loss of visual function (Duncan et al., $2007 b$ ), indicating that visual deprivation can have a potential effect on brain structure and function.

\section{Higher Visual Areas and Connection Between Visual Areas}

Many of the above-mentioned studies demonstrated the effect of glaucoma on higher visual areas, which are the secondary or associative areas of vision. Some of these studies showed a decreased functional activation after visual stimulation of the higher visual areas (Borges et al., 2015) and also a decreased cortical volume of associative areas (Bolacchi et al., 2012; Yu et al., 2013, 2014, 2015). The more plausible explanation of these changes is the transynaptic neurodegeneration. Other studies however found an increased functional activity in some higher visual areas (Jiang et al., 2017) and even an increased volume in some visual associative areas (Li et al., 2012; Chen W. W. et al., 2013; Williams et al., 2013; Jiang et al., 2017). Increased functional activity could be a result of reduced feedback signals from visual areas causing a reduction in GABA-mediated inhibitor signal (Chen et al., 2017). According to this latter hypothesis, visual area has an inhibition effect on some visual associative areas. Since the primary visual area is damaged in glaucoma, there is a reduction of inhibition that should cause a greater functional response to fellow eye input within the lesion projection zone. This was proved in murine (Crozier et al., 2007) and primate (Biswal et al., 2010) models but not in human eye (Boucard et al., 2007).Results of increased gray matter volume in visual association areas are more difficult to interpret. The increased volume $\mathrm{t}$ of these areas could be a sign of neuronal damage (microglia activation, neuronal swelling) or of increased cortical functionality and plasticity (increased arborization of dendrites and axons). The Jiang's study (Jiang et al., 2017) analyzed the same areas both in terms of volume with VBM and in terms of functional activity through BOLD level with the fMRI. Jiang et al. found that volume changes were not fully consistent with the regional blood flow changes; only frontal and parietal lobes have overlapped results. Increased volume in other areas without functional activation could be explained as the result of cell edema, sign of nerve damage.

Dai et al. (2013) found disrupted connectivity between the primary and higher visual cortex and between visual cortex and associative visual areas in the task-free state. Diminished connectivity between these areas may be associated with impairment in memory-related imagery, visual consolidation and integration of visual, auditory and tactile stimulation. In 
addition to areas of decreased connectivity, some brain regions of POAG patients showed increased connectivity with visual cortex. These changes could be related to recruitment to compensate areas of decreased activity or, alternatively, may be related to loss of inhibitor input.

Decreased connectivity in visual and working memory is confirmed by Frezzotti et al. study (Frezzotti et al., 2014) and it explains the impairment of object identification in POAG patients.

PACG patients share the same decreased visual information integration between the primary visual cortex and higher areas (Huang et al., 2015; Li et al., 2017).

\section{Areas Indirectly Involved in the Visual Pathway}

Several of the studies that this paper is based on reported alterations in areas not directly involved in the visual pathway. A study which measured the brain connectivity with the rs-fMRI in PACG patients (Cai et al., 2015) showed the increased connectivity in the anterior cingulate cortex (ACC) and the caudate, a component of the basal ganglia. The ACC is responsible for cognition and emotional control; its dysregulation in PACG patients could be caused by the higher prevalence of anxiety and depression in glaucomatous patients (Agorastos et al., 2013), even higher in PACG (Kong et al., 2015). The increased connectivity in the caudate is explained as a response to altered proprioception and somatosensory processing.

Decreased spontaneous activity in the claustrum and paracentral lobule lobe was observed in PACG patients using the same technique (rs-fMRI) (Chen et al., 2017). These finding may indicate impairment of the visuospatial function; the results of Zikou et al corroborate this hypothesis for the same reason (Zikou et al., 2012).

Alterations in extra-visual pathway areas are also demonstrated with structural examinations. A recent study using DTI (Boucard et al., 2016) demonstrated the degeneration of the corpus callosum in a cohort of normal tension glaucoma. This finding suggests the presence of neurodegeneration of the brain beyond what can be explained on the basis of propagated retinal and pre-geniculate damage. It is arduous to apply this conclusions to POAG patients; it is more reasonable that NTG has a different profile of neuronal cell death. Another area which showed volumetric alteration in glaucomatous patients was the amygdala that plays a role in the control of emotions (Wang et al., 2016).

In Frezzotti's study POAG patients showed gray matter atrophy in regions involved in cognitive processing (Frezzotti et al., 2014). In addition, several areas showed alteration in both Alzheimer disease and glaucoma: the hippocampus, involved in the memory process, the frontoorbital cortex (implied in the decision-making) and superior parietal lobule (special orientation).

While glaucoma normally manifest as a damage in main cerebral visual areas, there are also alterations possibly related to glaucoma in areas of the brain that are less known of. These parts of the brain for example are involved in other functions, emotional, decision making and memory.

\section{Possible Causes of Cortical Changes in Glaucoma Patients}

Mechanisms involved in cortical changes of glaucomatous patients remain debated. Also, the timing of the neurodegenerative cortical process is still to be unanimously ascertained. The main question is whether the cortical changes precede or follow the RGC neurodegeneration. Many studies supported the thesis that glaucomatous degeneration of the posterior visual pathway is a consequence of anterograde trans-synaptic diffusion of death signals triggered by RGC degeneration (Calkins and Horner, 2012). Bolacchi et al. (2012) analyzed the properties of the intraorbital optic nerve at two different levels: proximal and distal to the optic nerve head using the DTI. The study results indicate at early disease stage a significant decrease in connectivity in the proximal segment with respect to the distal site. Furthermore, reduced brain volume in visual-related cortex was found only in advanced stage glaucoma but not in early stage patients ( $\mathrm{Li}$ et al., 2012). Functional analysis through fMRI provided a detailed analysis of layer-specific neuronal signals in the subcortical visual nuclei (lateral geniculate nucleus and superior colliculus) and visual cortex. It emerges that earlystage glaucoma causes selective functional loss to large cells (responsive to $\mathrm{M}$ stimulus) in the lateral geniculate nucleus and superior colliculus, but not in the visual area (Zhang et al., 2016).

On the other hand, similarities between glaucoma and other neuro-degenerative diseases (including Alzheimer's disease) suggest a certain grade of retrograde trans-synaptic degeneration as well. A demonstration of this theory is the presence of damage also in areas not directly involved in the visual pathway (Cai et al., 2015; Boucard et al., 2016). In addition, functional changes in brain connectivity (Frezzotti et al., 2016; Murphy et al., 2016) together with reduced brain volume were found in early stage glaucoma, even before visual field damage (Murphy et al., 2016).

Vascular changes also have a possible role in the physiopathology as demonstrated by ischemic microinfarcts in the optic radiation and other cortical regions (Boucard et al., 2016).

\section{Correlation of Structural and Functional Parameters With Clinical Severity}

Many studies investigated the correlation between structural, functional and metabolic brain parameters and ophthalmic measurement such as cup-disc ratio, retinal nerve fiber layer thickness and visual field parameters (Hernowo et al., 2011; Nucci et al., 2012; Chen Z. et al., 2013; Michelson et al., 2013; Sidek et al., 2014; Cai et al., 2015; Gerente et al., 2015; Chen et al., 2017; Li et al., 2017). Many studies found a positive correlation with all these parameters, revealing that visual pathway damage correlates with clinical severity in glaucoma (Nucci et al., 2012; Chen Z. et al., 2013; Michelson et al., 2013; Sidek et al., 2014; Cai et al., 2015; Gerente et al., 2015; Chen et al., 2017).

The relationship with loss of visual field has important implications. In glaucoma a loss of $\sim 40-60 \%$ of the ganglion 
cells is already present when visual field defects are detectable by perimetry (Quigley et al., 1989; Harwerth et al., 1999, 2005; Kerrigan-Baumrind et al., 2000; Rolle et al., 2016). Structural neuroimaging techniques, such as DTI, are able to detect axonal injury of visual pathway at an early stage, even before visible visual field defect.

Moreover, a decreased cortical activity in the primary visual cortex is detected with fMRI in the cortical area corresponding to the central normal field (Qing et al., 2010). As previously reported, a big amount of RGC is lost before any abnormality in automated visual field testing is detectable. The remaining RGC undergo structural reorganization which consist in somata and dendritic arbors expansions. Consequently, cortical visual receptive filed increases in size. Thus, even if the central visual field is normal, the retina input is already reduced and the activity in visual cortex is reduced as well.

It is not surprising, that many authors propose neuroimaging techniques as a tool for earlier diagnosis of glaucoma in the future.

In regard to influence of glaucoma severity on brain changes, it is somehow difficult to provide a systematic analysis mainly because of differences in classification and parameters used to divide patients. Nevertheless, while functional connectivity was proved to be already altered in early POAG in both visual and nonvisual systems (Frezzotti et al., 2016; Zhang et al., 2016), structural changes (mainly gray matter atrophy) was unanimously found in more advanced/severe POAG, but not in all early POAG analyzed (Li et al., 2012; Zhang et al., 2015). Studies investigating cortical geometry in mild and severe POAG demonstrated a progressively aggravated degeneration with the increasing severity of disease. Cortical degeneration occurs not only in visual cortex (both V1 and V2), but also in the middle temporal visual area (V5/MT+), which is thought to be specialized for processing motion (Yu et al., 2014, 2015).

Overall, structural, functional, and metabolic brain changes show a good correlation with clinical measurement of severity.

\section{Neuronal Plasticity}

Many human studies including those of this review have suggested that even during adulthood the visual cortex maintains the ability to structurally and functionally reorganize (Rosa et al., 2013). Visual cortical plasticity was also proved in healthy subjects after visual training (Berry and Nedivi, 2016). Neuroplasticity can be both structural, which implies changes in axons, and functional (Rosa et al., 2013). Functional reorganization is related to the unmasking of intracortical feedback to early visual cortex from higher visual areas (Crozier et al., 2007; Biswal et al., 2010).

Postoperative enhancement in neuronal connectivity was demonstrated in a group of PACG patients 3 months after surgery with normalization of IOP (Cai et al., 2015). The areas that showed a better connectivity were visual cortex, primary, sensory, and supplementary motor areas Further studies (Li et al., 2017) demonstrated functional restoration after glaucomatous surgery and IOP normalization. One possible explanation for this result is the post-operative plasticity in functional networks.
These changes can occur 3 months after the surgery, and it is possible that they are due to restoration process. Li et al. found a decreased connectivity of the visual area with the resting statefMRI after surgery and IOP normalization (Li et al., 2017). The decreased connectivity of the visual area reflects the decreased compensation of other brain areas following the reduction of IOP and improving vision.

On the contrary, some authors suggested a remarkable level of stability within the adult primary and extrastriate visual cortex, without any evidence of neurodegeneration affecting cells receiving visual information of fellow eye in patients with unilateral glaucoma (Borges et al., 2015).

\section{Limits and Suggestion for Future Studies}

Our study suffers from several limitations mainly due to limitations associated with experimental design and available tools of studies. First, different brain atlases were used to annotate localization of cortical areas. Moreover, in regard to the fMRI studies, differences in type, frequency, size, brightness, and color of stimuli are present in different studies. Thus, cortical activity is certainly influenced by the stimulus which causes conclusions of studies to be partially conflicting with each other. Another limitation consists in the heterogeneity of age of patients, severity of damage and duration of disease between the studies.

Some suggestion for future studies include a consistent number of both glaucomatous and healthy subject, division of glaucomatous patients in severity group through well-established classification (i.e., Hodapp criteria), application of structural, functional and metabolic methods. Correspondence of structural and functional-metabolic abnormalities gives strength to results.

\section{Therapeutic Perspectives}

The identification of neurodegeneration in the visual centers of the brain has important implications for the treatment of glaucoma. Emerging therapeutic interventions, such as RGC transplantation and artificial retinal implants (Mathieson et al., 2012; Venugopalan et al., 2016; Jutley et al., 2017; Sena and Lindsley, 2017), need to consider the degree of neurodegeneration in the posterior visual pathway as a confounding factor in the efficacy of therapeutic interventions. Glaucomatous neurodegeneration in the brain also has implications for assessing the efficacy of neuroprotection, suggesting that the focus of these therapies should be extended to include the whole brain. Furthermore, abnormalities of the brain should be included as endpoints in studies of therapeutic efficacy in glaucoma trials.

\section{CONCLUSION}

Systematic review yields the advantage of aggregating information and more robust estimates than individual studies. This approach can be particularly useful when evaluating new procedures with relatively small studies. Brain changes in glaucomatous subjects have been proved with autoptic and experimental studies in the past. Recently, the advent 
of non-invasive neuroimaging has led to a growth in studies investigating the brain damage of glaucomatous patients.

The results of this meta-analysis show functional and structural changes throughout the visual and nonvisual pathway in glaucomatous patients. These changes correlate with clinical findings and severity of the disease. Entity and mechanism of cortical reorganization are still not completely clear.

This review contributes to the better understanding of brain abnormalities in glaucoma. The results may stimulate further speculation about brain plasticity at a later age and therapeutic strategies, such as the prevention of cortical degeneration in patients with glaucoma.

\section{REFERENCES}

Agorastos, A., Skevas, C., Matthaei, M., Otte, C., Klemm, M., Richard, G., et al. (2013). Depression, anxiety, and disturbed sleep in glaucoma. J. Neuropsychiatry Clin. Neurosci. 25, 205-213. doi: 10.1176/appi.neuropsych.12020030

Alexander, A. L., Lee, J. E., Lazar, M., and Field, A. S. (2007). Diffusion tensor imaging of the brain. Neurotherapeutics 4, 316-329. doi: 10.1016/j.nurt.2007.05.011

Ashburner, J., and Friston, K. J. (2000). Voxel-based morphometry-the methods. Neuroimage 11, 805-821. doi: 10.1006/nimg.2000.0582

Bayer, A. U., and Ferrari, F. (2002). Severe progression of glaucomatous optic neuropathy in patients with Alzheimer's disease. Eye 16, 209-212. doi: 10.1038/sj.eye.6700034

Bayer, A. U., Ferrari, F., and Erb, C. (2002). High occurrence rate of glaucoma among patients with Alzheimer's disease. Eur. Neurol. 47, 165-168. doi: 10.1159/000047976

Berry, K. P., and Nedivi, E. (2016). Experience-dependent structural plasticity in the visual system. Annu. Rev. Vis. Sci. 2, 17-35. doi: 10.1146/annurev-vision-111815-114638

Biswal, B. B., Mennes, M., Zuo, X. N., Gohel, S., Kelly, C., Smith, S. M., et al. (2010). Toward discovery science of human brain function. Proc. Natl. Acad. Sci. U.S.A. 107, 4734-4739. doi: 10.1073/pnas.0911855107

Bogorodzki, P., Piatkowska-Janko, E., Szaflik, J., Szaflik, J. P., Gacek, M., and Grieb, P. (2014). Mapping cortical thickness of the patients with unilateral end-stage open angle glaucoma on planar cerebral cortex maps. PLoS ONE 9:e93682. doi: 10.1371/journal.pone.0093682

Bolacchi, F., Garaci, F. G., Martucci, A., Meschini, A., Fornari, M., Marziali, S., et al. (2012). Differences between proximal versus distal intraorbital optic nerve diffusion tensor magnetic resonance imaging properties in glaucoma patients. Invest. Ophthalmol. Vis. Sci. 53, 4191-4196. doi: 10.1167/iovs. 11-9345

Borges, V. M., Danesh-Meyer, H. V., Black, J. M., and Thompson, B. (2015). Functional effects of unilateral open-angle glaucoma on the primary and extrastriate visual cortex. J. Vis. 15:9. doi: 10.1167/15.15.9

Boucard, C. C., Hanekamp, S., Curčić-Blake, B., Ida, M., Yoshida, M., and Cornelissen, F. W. (2016). Neurodegeneration beyond the primary visual pathways in a population with a high incidence of normal-pressure glaucoma. Ophthalmic. Physiol. Opt. 36, 344-353. doi: 10.1111/opo.12297

Boucard, C. C., Hernowo, A. T., Maguire, R. P., Jansonius, N. M., Roerdink, J. B., Hooymans, J. M., et al. (2009). Changes in cortical grey matter density associated with long-standing retinal visual field defects. Brain 132, 1898-1906. doi: 10.1093/brain/awp119

Boucard, C. C., Hoogduin, J. M., van der Grond, J., and Cornelissen, F. W. (2007). Occipital proton magnetic resonance spectroscopy (1H-MRS) reveals normal metabolite concentrations in retinal visual field defects. PLOS ONE 2:e222. doi: 10.1371/journal.pone.0000222

Brown, H. D., Woodall, R. L., Kitching, R. E., Baseler, H. A., and Morland, A. B. (2016). Using magnetic resonance imaging to assess visual deficits: a review. Ophthalmic. Physiol. Opt. 36, 240-265. doi: 10.1111/opo.12293

\section{AUTHOR CONTRIBUTIONS}

RN conception and design of study. LD acquisition and analysis of data. LD and TR drafting the manuscript. RN, LD, and TR revising the manuscript critically for important intellectual content. RN, LD, and TR approval of the version of the manuscript to be published.

\section{SUPPLEMENTARY MATERIAL}

The Supplementary Material for this article can be found online at: https://www.frontiersin.org/articles/10.3389/fnins. 2018.00363/full\#supplementary-material

Cai, F., Gao, L., Gong, H., Jiang, F., Pei, C., Zhang, X., et al. (2015). Network centrality of resting-state fMRI in primary angle-closure glaucoma before and after surgery. PLoS ONE 10:e0141389. doi: 10.1371/journal.pone.0141389

Calkins, D. J., and Horner, P. J. (2012). The cell and molecular biology of glaucoma: axonopathy and the brain. Invest. Ophthalmol. Vis. Sci. 53, 2482-2484. doi: $10.1167 /$ iovs.12-9483i

Chang, E. E., and Goldberg, J. L. (2012). Glaucoma 2.0: neuroprotection, neuroregeneration, neuroenhancement. Ophthalmology 119, 979-986. doi: 10.1016/j.ophtha.2011.11.003

Chen, W. W., Wang, N., Cai, S., Fang, Z., Yu, M., Wu, Q., et al. (2013). Structural brain abnormalities in patients with primary open-angle glaucoma: a study with 3T MR imaging. Invest. Ophthalmol. Vis. Sci. 54, 545-554. doi: 10.1167/iovs.12-9893

Chen, W., Zhang, L., Xu, Y. G., Zhu, K., and Luo, M. (2017). Primary angle-closure glaucomas disturb regional spontaneous brain activity in the visual pathway: an fMRI study. Neuropsychiatr. Dis. Treat. 13, 1409-1417. doi: 10.2147/NDT.S134258

Chen, Z., Lin, F., Wang, J., Li, Z., Dai, H., Mu, K., et al. (2013). Diffusion tensor magnetic resonance imaging reveals visual pathway damage that correlates with clinical severity in glaucoma. Clin. Exp. Ophthalmol. 1, 43-49. doi: 10.1111/j.1442-9071.2012.02832.x

Clarkson, M. J., Cardoso, M. J., Ridgway, G. R., Modat, M., Leung, K. K., Rohrer, J. D., et al. (2011). A comparison of voxel and surface based cortical thickness estimation methods. Neuroimage 57, 856-865. doi: 10.1016/j.neuroimage.2011.05.053

Crozier, R. A., Wang, Y., Liu, C. H., and Bear, M. F. (2007). Deprivationinduced synaptic depression by distinct mechanisms in different layers of mouse visual cortex. Proc. Natl. Acad. Sci. U.S.A. 104, 1383-1388. doi: 10.1073/pnas.0609596104

Dai, H., Morelli, J. N., Ai, F., Yin, D., Hu, C., Xu, D., et al. (2013). Restingstate functional MRI: functional connectivity analysis of the visual cortex in primary open-angle glaucoma patients. Hum. Brain Mapp. 34, 2455-2463. doi: 10.1002/hbm.22079

Davis, B. M., Crawley, L., Pahlitzsch, M., Javaid, F., and Cordeiro, M. F. (2016). Glaucoma: the retina and beyond. Acta Neuropathol. 132, 807-826. doi: 10.1007/s00401-016-1609-2

Duncan, R. O., Sample, P. A., Weinreb, R. N., Bowd, C., and Zangwill, L. M. (2007a). Retinotopic organization of primary visual cortex in glaucoma: a method for comparing cortical function with damage to the optic disk. Invest. Ophthalmol. Vis. Sci. 48, 733-744. doi: 10.1167/iovs.06-0773

Duncan, R. O., Sample, P. A., Weinreb, R. N., Bowd, C., and Zangwill, L. M. (2007b). Retinotopic organization of primary visual cortex in glaucoma: comparing fMRI measurements of cortical function with visual field loss. Prog. Retin. Eye Res. 26, 38-56. doi: 10.1016/j.preteyeres.2006.10.001

El-Rafei, A., Engelhorn, T., Wärntges, S., Dörfler, A., Hornegger, J., and Michelson, G. (2011). A framework for voxel-based morphometric analysis of the optic radiation using diffusion tensor imaging in glaucoma. Magn. Reson. Imaging 29, 1076-1087. doi: 10.1016/j.mri.2011.02.034

El-Rafei, A., Engelhorn, T., Wärntges, S., Dörfler, A., Hornegger, J., and Michelson, G. (2013). Glaucoma classification based on visual pathway 
analysis using diffusion tensor imaging. Magn. Reson. Imaging 31, 1081-1091. doi: 10.1016/j.mri.2013.01.001

Engelhorn, T., Michelson, G., Waerntges, S., Struffert, T., Haider, S., and Doerfler, A. (2011). Diffusion tensor imaging detects rarefaction of optic radiation in glaucoma patients. Acad. Radiol. 18, 764-769. doi: 10.1016/j.acra.2011.01.014

Fiedorowicz, M., Dyda, W., Rejdak, R., and Grieb, P. (2011). Magnetic resonance in studies of glaucoma. Med. Sci. Monit. 17, RA227-R232. doi: 10.12659/MSM.881973

Frezzotti, P., Giorgio, A., Motolese, I., De Leucio, A., Iester, M., Motolese, E., et al. (2014). Structural and functional brain changes beyond visual system in patients with advanced glaucoma. PLoS ONE 9:e105931. doi: 10.1371/journal.pone.0105931

Frezzotti, P., Giorgio, A., Toto, F., De Leucio, A., and De Stefano, N. (2016). Early changes of brain connectivity in primary open angle glaucoma. Hum. Brain Mapp. 37, 4581-4596. doi: 10.1002/hbm.23330

Garaci, F. G., Bolacchi, F., Cerulli, A., Melis, M., Spanò A., Cedrone, C., et al. (2009). Optic nerve and optic radiation neurodegeneration in patients with glaucoma: in vivo analysis with 3-T diffusion-tensor MR imaging. Radiology 252, 496-501. doi: 10.1148/radiol.2522081240

Gerente, V. M., Schor, R. R., Chaim, K. T., Felix Mde, M., Ventura, D. F., Teixeira, S. H., et al. (2015). Evaluation of glaucomatous damage via functional magnetic resonance imaging, and correlations thereof with anatomical and psychophysical ocular findings. PLOS ONE 10:e0126362. doi: 10.1371/journal.pone.0126362

Giorgio, A., Zhang, J., Costantino, F., De Stefano, N., and Frezzotti, P. (2018). Diffuse brain damage in normal tension glaucoma. Hum. Brain Mapp. 39, 532-541. doi: 10.1002/hbm.23862

Gupta, N., and Yücel, Y. H. (2003). Brain changes in glaucoma. Eur. J. Ophthalmol. 3, S32-S35. doi: 10.1177/112067210301303S06

Gupta, N., Ang, L.-C. C., Noel de Tilly, L., Bidaisee, L., Yucel, Y. H., Noël de Tilly, L., et al. (2006). Human glaucoma and neural degeneration in intracranial optic nerve, lateral geniculate nucleus, and visual cortex. Br. J. Ophthalmol. 90, 674-678. doi: 10.1136/bjo.2005.086769

Gupta, N., Yücel, Y. H., and Yucel, Y. H. (2007). Glaucoma as a neurodegenerative disease. Curr. Opin. Ophthalmol. 18, 110-114 doi: 10.1097/ICU.0b013e3280895aea

Harwerth, R. S., Carter-Dawson, L., Shen, F., Smith, E. L. III., and Crawford, M. L. (1999). Ganglion cell losses underlying visual field defects from experimental glaucoma. Invest. Ophthalmol. Vis. Sci. 40, 2242-2250.

Harwerth, R. S., Carter-Dawson, L., Smith, E. L., and Crawford, M. L. J. (2005). Scaling the structure-function relationship for clinical perimetry. Acta Ophthalmol. Scand. 83, 448-455. doi: 10.1111/j.1395-3907.2005.00494.x

Hernowo, A. T., Boucard, C. C., Jansonius, N. M., Hooymans, J. M., and Cornelissen, F. W. (2011). Automated morphometry of the visual pathway in primary open-angle glaucoma. Invest. Ophthalmol. Vis. Sci. 52, 2758-2766. doi: $10.1167 /$ iovs.10-5682

Huang, X., Zhong, Y. L., Zeng, X. J., Zhou, F., Liu, X. H., Hu, P. H., et al. (2015). Disturbed spontaneous brain activity pattern in patients with primary angleclosure glaucoma using amplitude of low-frequency fluctuation: a fMRI study. Neuropsychiatr. Dis. Treat. 11, 1877-1883. doi: 10.2147/NDT.S87596

Jiang, M. M., Zhou, Q., Liu, X. Y., Shi, C. Z., Chen, J., and Huang, X. H. (2017). Structural and functional brain changes in early- and mid-stage primary open-angle glaucoma using voxel-based morphometry and functional magnetic resonance imaging. Medicine 96:e6139. doi: 10.1097/MD.0000000000006139

Jutley, G., Luk, S. M., Dehabadi, M. H., and Cordeiro, M. F. (2017). Management of glaucoma as a neurodegenerative disease. Neurodegener. Dis. Manag. 7, 157-172. doi: 10.2217/nmt-2017-0004

Kapetanakis, V. V., Chan, M. P., Foster, P. J., Cook, D. G., Owen, C. G., and Rudnicka, A. R. (2016). Global variations and time trends in the prevalence of primary open angle glaucoma (POAG): a systematic review and meta-analysis. Br. J. Ophthalmol. 100, 86-93. doi: 10.1136/bjophthalmol-2015-307223

Kaushik, M., Graham, S. L., Wang, C., and Klistorner, A. (2014). A topographical relationship between visual field defects and optic radiation changes in glaucoma. Invest. Ophthalmol. Vis. Sci. 55, 5770-5775. doi: $10.1167 /$ iovs. $14-14733$

Kerrigan-Baumrind, L. A., Quigley, H. A., Pease, M. E., Kerrigan, D. F., and Mitchell, R. S. (2000). Number of ganglion cells in glaucoma eyesccompared with threshold visual field tests in the same persons. Invest. Ophthalmol. Vis. Sci. 41, 741-748

Kollias, S. S. (2004). Investigations of the human visual system using functional magnetic resonance imaging (FMRI). Eur. J. Radiol. 49, 64-75. doi: 10.1016/j.ejrad.2003.09.005

Kong, X., Yan, M., Sun, X., Xiao, Z. (2015). Anxiety and depression are more prevalent in primary angle closure glaucoma than in primary open-angle glaucoma. J. Glaucoma 24, e57-e63. doi: 10.1097/IJG.0000000000000025

Lestak, J., Tintera, J., Svata, Z., Ettler, L., and Rozsival, P. (2014). Glaucoma and CNS. comparison of fMRI results in high tension and normal tension glaucoma. Biomed. Pap. Med. Fac. Univ. Palacky. Olomouc Czech Repub. 158, 144-153. doi: $10.5507 / \mathrm{bp} .2013 .038$

Li, C., Cai, P., Shi, L., Lin, Y., Zhang, J., Liu, S., et al. (2012). Voxel-based morphometry of the visual-related cortex in primary open angle glaucoma. Curr. Eye Res. 37, 794-802. doi: 10.3109/02713683.2012.683506

Li, S., Li, P., Gong, H., Jiang, F., Liu, D., Cai, F., et al. (2017). Intrinsic functional connectivity alterations of the primary visual cortex in primary angle-closure glaucoma patients before and after surgery: a resting-state fMRI study. PLoS ONE 12:e0170598. doi: 10.1371/journal.pone.0170598

Li, T., Liu, Z., Li, J., Liu, Z., Tang, Z., Xie, X., et al. (2014). Altered amplitude of low-frequency fluctuation 656 in primary open-angle glaucoma: a resting-state FMRI study. Invest. Ophthalmol. Vis. Sci. 56, 322-329. doi: $10.1167 /$ iovs.14-14974

Lu, P., Shi, L., Du, H., Xie, B., Li, C., Li, S., et al. (2013). Reduced white matter integrity in primary open-angle glaucoma: a DTI study using tract-based spatial statistics. J. Neuroradiol. 40, 89-93. doi: 10.1016/j.neurad.2012.04.001

Mastropasqua, R., Agnifili, L., Mattei, P. A., Caulo, M., Fasanella, V., Navarra, R., et al. (2015). Advanced morphological and functional magnetic resonance techniques in glaucoma. Biomed. Res. Int. 2015:160454. doi: $10.1155 / 2015 / 160454$

Mathieson, K., Loudin, J., Goetz, G., Huie, P., Wang, L., Kamins, T. I., et al. (2012). Photovoltaic retinal prosthesis with high pixel density. Nat. Photonics 6, 391-397. doi: 10.1038/nphoton.2012.104

McKinnon, S. J. (2012). The cell and molecular biology of glaucoma: common neurodegenerative pathways and relevance to glaucoma. Invest. Ophthalmol. Vis. Sci. 53, 2485-2487. doi: 10.1167/iovs.12-9483j

Michelson, G., Engelhorn, T., Wärntges, S., El Rafei, A., Hornegger, J., and Doerfler, A. (2013). DTI parameters of axonal integrity and demyelination of the optic radiation correlate with glaucoma indices. Graefes Arch. Clin. Exp. Ophthalmol. 251, 243-253. doi: 10.1007/s00417-011-1887-2

Miki, A., Haselgrove, J. C., and Liu, G. T. (2002). Functional magnetic resonance imaging and its clinical utility in patients with visual disturbances. Surv. Ophthalmol. 47, 562-579. doi: 10.1016/S0039-6257(02)00356-9

Miki, A., Liu, G. T., Modestino, E. J., Liu, C. S., Bonhomme, G. R., Dobre, C. M., et al. (2001). Functional magnetic resonance imaging of the visual system. Curr. Opin. Ophthalmol. 12, 423-431. doi: 10.1097/00055735-200112000-00007

Murai, H., Suzuki, Y., Kiyosawa, M., Tokumaru, A. M., Ishiwata, K., and Ishii, K. (2015). Cerebral glucose metabolism in the striate cortex positively correlates with fractional anisotropy values of the optic radiation in patients with glaucoma. Clin. Exp. Ophthalmol. 43, 711-719. doi: 10.1111/ceo.12543

Murphy, M. C., Conner, I. P., Teng, C. Y., Lawrence, J. D., Safiullah Z1, Wang, B., et al. (2016). Retinal structures and visual cortex activity are impaired prior to clinical vision loss in glaucoma. Sci. Rep. 6:31464. doi: 10.1038/srep31464

Nucci, C., Mancino, R., Martucci, A., Bolacchi, F., Manenti, G., et al. (2012). 3-T Diffusion tensor imaging of the optic nerve in subjects with glaucoma: correlation with GDx-VCC, HRT-III and Stratus optical coherence tomography findings. Br. J. Ophthalmol. 96, 976-980. doi: 10.1136/bjophthalmol-2011-301280

Omodaka, K., Murata, T., Sato, S., Takahashi, M., Tatewaki, Y., Nagasaka, T., et al. (2014). Correlation of magnetic resonance imaging optic nerve parameters to optical coherence tomography and the visual field in glaucoma. Clin. Exp. Ophthalmol. 42, 360-368. doi: 10.1111/ceo.12237

Prins, D., Hanekamp, S., and Cornelissen, F. W. (2016). Structural brain MRI studies in eye diseases: are they clinically relevant? Rev. Curr. Findings Acta Ophthalmol. 94, 113-121. doi: 10.1111/aos.12825

Qing, G., Zhang, S., Wang, B., and Wang, N. (2010). Functional MRI signal changes in primary visual cortex corresponding to the central normal visual 
field of patients with primary open-angle glaucoma. Invest. Ophthalmol. Vis. Sci. 51, 4627-4634. doi: 10.1167/iovs.09-4834

Quigley, H. A., Dunkelberger, G. R., and Green, W. R. (1989). Retinal ganglion cell atrophy correlated with automated perimetry in human eyes with glaucoma. Am. J. Ophthalmol. 107, 453-464. doi: 10.1016/0002-9394(89)90488-1

Rolle, T., Dallorto, L., and Bonetti, B. (2016). Retinal and macular ganglion cell count estimated with optical coherence tomography RTVUE-100 as a candidate biomarker for glaucoma. Invest. Ophthalmol. Vis. Sci. 57, 5772-5779. doi: $10.1167 /$ iovs. $15-18882$

Rosa, A. M., Silva, M. F., Ferreira, S., Murta, J., and Castelo-Branco, M. (2013). Plasticity in the human visual cortex: an ophthalmology-based perspective. Biomed. Res. Int. 2013, 1-13. doi: 10.1155/2013/568354

Schoemann, J., Engelhorn, T., Waerntges, S., Doerfler, A., El-Rafei, A., and Michelson, G. (2014). Cerebral microinfarcts in primary open-angle glaucoma correlated with DTI-derived integrity of optic radiation. Invest. Ophthalmol. Vis. Sci. 55, 7241-7247. doi: 10.1167/iovs.14-14919

Sena, D. F., and Lindsley, K. (2017). Neuroprotection for treatment of glaucoma in adults. Cochrane Database Syst. Rev. 25:CD006539. doi: 10.1002/14651858.CD006539.pub4

Sidek, S., Ramli, N., Rahmat, K., Ramli, N. M., Abdulrahman, F., and Tan, L. K. (2014). Glaucoma severity affects diffusion tensor imaging (DTI) parameters of the optic nerve and optic radiation. Eur. J. Radiol. 83, 1437-1441. doi: 10.1016/j.ejrad.2014.05.014

Smitha, K. A., Akhil Raja, K., Arun, K. M., Rajesh, P. G., Thomas, B., Kapilamoorthy, T. R., et al. (2017). Resting state fMRI: a review on methods in resting state connectivity analysis and resting state networks. Neuroradiol. J. 30, 305-317. doi: 10.1177/1971400917697342

Song, X., Wang, G., Zhang, T., Feng, L., An, P., and Zhu, Y. (2012). Functional magnetic resonance imaging evaluation of visual cortex activation in patients with anterior visual pathway lesions. Neural Regen. Res. 7, 692-696. doi: 10.3969/j.issn.1673-5374.2012.09.009

Song, Y., Mu, K., Wang, J., Lin, F., Chen, Z., Yan, X., et al. (2014). Altered spontaneous brain activity in primary open angle glaucoma: a restingstate functional magnetic resonance imaging study. PLoS ONE 9:e89493. doi: 10.1371/journal.pone.0089493

Tamura, H., Kawakami, H., Kanamoto, T., Kato, T., Yokoyama, T., Sasaki, K., et al. (2006). High frequency of open-angle glaucoma in Japanese patients with Alzheimer's disease. J. Neurol. Sci. 246, 79-83. doi: 10.1016/j.jns.2006.02.009

Tellouck, L., Durieux, M., Coupé P., Cougnard-Grégoire, A., Tellouck, J., Tourdias, T., et al. (2016). Optic radiations microstructural changes in glaucoma and association with severity: a study using 3tesla-magnetic resonance diffusion tensor imaging. Invest. Ophthalmol. Vis. Sci. 57, 6539-6547. doi: $10.1167 /$ iovs.16-19838

Tham, Y.-C. C., Li, X., Wong, T. Y., Quigley, H. A., Aung, T., Ed, F., et al. (2014). Global prevalence of glaucoma and projections of glaucoma burden through 2040. Syst. Rev. Meta Analysis Ophthalmol. 121, 2081-2090. doi: 10.1016/j.ophtha.2014.05.013

Venugopalan, P., Wang, Y., Nguyen, T., Huang, A., Muller, K. J., and Goldberg, J. L. (2016). Transplanted neurons integrate into adult retinas and respond to light. Nat. Commun. 7:10472. doi: 10.1038/ncomms10472

Wang, J., Li, T., Sabel, B. A., Chen, Z., Wen, H., Li, J., et al. (2016). Structural brain alterations in primary open angle glaucoma: a 3T MRI study. Sci. Rep. 6:18969. doi: $10.1038 /$ srep 18969

Wang, J., Li, T., Wang, N., Xian, J., and He, H. (2016a). Graph theoretical analysis reveals the reorganization of the brain network pattern in primary open angle glaucoma patients. Eur. Radiol. 26, 3957-3967. doi: 10.1007/s00330-016-4221-x

Wang, J., Li, T., Zhou, P., Wang, N., Xian, J., and He, H. (2016b). Altered functional connectivity within and between the default model network and the visual network in primary open-angle glaucoma: a resting-state fMRI study. Brain Imaging Behav. 11, 1154-1163. doi: 10.1007/s11682-016-9597-3.

Wang, M. Y., Wu, K., Xu, J. M., Dai, J., Qin, W., Liu, J., et al. (2013). Quantitative 3-T diffusion tensor imaging in detecting optic nerve degeneration in patients with glaucoma: association with retinal nerve fiber layer thickness and clinical severity. Neuroradiology 55, 493-498. doi: 10.1007/s00234-013-1133-1

Weinreb, R. N., Aung, T., and Medeiros, F. A. (2014). The pathophysiology and treatment of glaucoma: a review. JAMA 311, 1901-1911. doi: 10.1001 /jama.2014.3192
Williams, A. L., Lackey, J., Wizov, S. S., Chia, T. M., Gatla, S., Moster, M. L., et al. (2013). Evidence for widespread structural brain changes in glaucoma: a preliminary voxel-based MRI study. Invest. Ophthalmol. Vis. Sci. 54, 5880-5887. doi: $10.1167 /$ iovs.13-11776

Wostyn, P., Audenaert, K., and De Deyn, P. P. (2009). Alzheimer's disease and glaucoma: is there a causal relationship? Br. J. Ophthalmol. 93, 1557-1559. doi: 10.1136/bjo.2008.148064

Wostyn, P., Audenaert, K., and De Deyn, P. P. (2010). Alzheimer's disease: cerebral glaucoma? Med. Hypotheses 74, 973-977 doi: 10.1016/j.mehy.2009.12.019

Yu, L., Xie, B., Yin, X., Liang, M., Evans, A. C., Wang, J., et al. (2013). Reduced cortical thickness in primary open-angle glaucoma and its relationship to the retinal nerve fiber layer thickness. PLOS ONE 8:e73208. doi: 10.1371/journal.pone.0073208

Yu, L., Xie, L., Dai, C., Xie, B., Liang, M., Zhao, L., et al. (2015). Progressive thinning of visual cortex in primary open-angle glaucoma of varying severity. PLOS ONE 10:e0121960. doi: 10.1371/journal.pone.0121960

Yu, L., Yin, X., Dai, C., Liang, M., Wei, L., Li, C., et al. (2014). Morphologic changes in the anterior and posterior subregions of V1 and V2 and the V5/MT+ in patients with primary open-angle glaucoma. Brain Res. 1588, 135-143. doi: 10.1016/j.brainres.2014.09.005

Yücel, Y. H., Zhang, Q., Gupta, N., Kaufman, P. L., and Weinreb, R. N. (2000). Loss of neurons in magnocellular and parvocellular layers of the lateral geniculate nucleus in glaucoma. Arch. Ophthalmol. 118, 378-384. doi: 10.1001/archopht.118.3.378

Yücel, Y. H., Zhang, Q., Weinreb, R. N., Kaufman, P. L., and Gupta, N. (2001). Atrophy of relay neurons in magno- and parvocellular layers in the lateral geniculate nucleus in experimental glaucoma. Invest. Ophthalmol. Vis. Sci. 42, 3216-3222

Zhang, P., Wen, W., Sun, X., and He, S. (2016). Selective reduction of fMRI responses to transient achromatic stimuli in the magnocellular layers of the LGN and the superficial layer of the SC of early glaucoma patients. Hum. Brain Mapp. 37, 558-569. doi: 10.1002/hbm.23049

Zhang, S., Wang, B., Xie, Y., Zhu, S., Thomas, R., Qing, G., et al. (2015). Retinotopic changes in the gray matter volume and cerebral blood flow in the primary visual cortex of patients with primary open-angle glaucoma. Invest. Ophthalmol. Vis. Sci. 56, 6171-6178. doi: 10.1167/iovs.15-17286

Zhang, Y. Q., Li, J., Xu, L., Zhang, L., Wang, Z. C., Yang, H., et al. (2012). Anterior visual pathway assessment by magnetic resonance imaging in normal-pressure glaucoma. Acta Ophthalmol. 90, e295-e302. doi: $10.1111 / \mathrm{j} .1755-3768.2011 .02346 . \mathrm{x}$

Zhang, Y., Chen, X., Wen, G., Wu, G., and Zhang, X. (2013). Proton magnetic resonance spectroscopy [(1)H-MRS] reveals geniculocalcarine and striate area degeneration in primary glaucoma. PLoS ONE 8:e73197. doi: 10.1371/journal.pone.0073197

Zhou, W., Muir, E. R., Chalfin, S., Nagi, K. S., and Duong, T. Q. (2017a). MRI study of the posterior visual pathways in primary open angle glaucoma. J. Glaucoma 26, 173-181. doi: 10.1097/IJG.0000000000000558

Zhou, W., Muir, E. R., Nagi, K. S., Chalfin, S., Rodriguez, P., and Duong, T. Q. (2017b). Retinotopic fMRI reveals visual dysfunction and functional reorganization in the visual cortex of mild to moderate glaucoma patients. J. Glaucoma 26, 430-437. doi: 10.1097/IJG.0000000000000641

Zikou, A. K., Kitsos, G., Tzarouchi, L. C., Astrakas, L., Alexiou, G. A., and Argyropoulou, M. I. (2012). Voxel-based morphometry and diffusion tensor imaging of the optic pathway in primary open-angle glaucoma: a preliminary study. Am. J. Neuroradiol. 33, 128-134. doi: 10.3174/ajnr.A2714

Conflict of Interest Statement: The authors declare that the research was conducted in the absence of any commercial or financial relationships that could be construed as a potential conflict of interest.

Copyright (C) 2018 Nuzzi, Dallorto and Rolle. This is an open-access article distributed under the terms of the Creative Commons Attribution License (CC $B Y)$. The use, distribution or reproduction in other forums is permitted, provided the original author(s) and the copyright owner are credited and that the original publication in this journal is cited, in accordance with accepted academic practice. No use, distribution or reproduction is permitted which does not comply with these terms. 